\title{
Das echte Potenzial der Wochenblister
}

\section{Eine Untersuchung zu Einsatzpotenzial, Versorgungs- nutzen und wirtschaftlichen Effekten für Patienten, Kostenträger und Gesundheitssystem anhand von GKV- Routinedaten}

Im Krankenkassenwettbewerb werden vor dem Hintergrund des nahenden Gesundheitsfonds relative Kostenvorteile in der Chronikerversorgung erfolgsentscheidend. Entsprechend werden die Kostenträger u.a von den Leistungserbringern kosteneffektive Therapiekonzepte auch für die voraussichtlich 50 bis $80^{1}$ ausgleichsrelevanten Krankheiten einfordern. Damit rücken ebenfalls die Arzneimitteltherapiekosten in den Mittelpunkt.

Vor diesem Hintergrund wird zunehmend das Potenzial der „patientenindividuellen Arzneimittelversorgung“ diskutiert. Im Fokus steht dabei das Wochenblisterkonzept für den ambulanten Sektor. Belastbare Aussagen über Relevanz und Nutzen von Wochenblistern liegen für Deutschland nur sehr eingeschränkt vor.

Wegen der im Vollversorgungsansatz „Gesundes Kinzigtal“ ausgeübten Praxis des „Einsparcontracting“ ist neben der Krankenkasse auch die entsprechende Managementgesellschaft daran interessiert, die Arzneimitteltherapie zu optimieren. Um das Potenzial von Wochenblistern zu ermitteln, wurde eine auf GKV - Routinedaten von ca. 30.000 Versicherten beruhende Analyse durchgeführt. Dabei wurde festgestellt, dass das Potenzial von Wochenblistern vor allem in der koordinierten Gesamtmedikation älterer multimorbider Chroniker besteht und deutlich größer ausfällt als erwartet. Aufgrund definierter Kriterien wurde festgestellt, dass nicht jedes Wochenblisterkonzept automatisch zu dem erwarteten Mehrnutzen führt. Es wird konkret dargestellt, welche Anforderungen an ein Wochenblisterkonzept zu stellen sind und wie es in die Funktionsmechanismen des deutschen Gesundheitssystems einzubinden ist, um das mit der patientenindividuellen Arzneimittelversorgung verbundene Potenzial zu realisieren. Dabei wird der Schwerpunkt auf die Kosten-Nutzen Perspektive der Kostenträger gelegt und konkret darauf eingegangen, in welcher Weise diese von einem entsprechend ausgearbeiteten Wochenblisterkonzept profitieren können.

\section{Magnus Stüve, Helmut Hildebrandt, Gisela Daul, Jack Salmon}

\section{Einleitung/ Hintergrund}

Die patientenindividuelle Arzneimittelversorgung (PAV) und die damit verbundene Verblisterung von Arzneimitteln ist in Deutschland seit Inkrafttreten der 14. Novelle des

Stüve, Magnus, Senior Consultant, Hildebrandt GesundheitsConsult GmbH;

Hildebrandt, Helmut, Geschäftsführer der Managementgesellschaft "Gesundes Kinzigtal GmbH“;

Daul, Gisela, , Referatsleiterin Neue Versorgungsformen AOK Baden-Württemberg;

Salmon, Jack, Ph.D., Professor, Pharmacy Administration, College of Pharmacy; Health Policy \& Administration, School of Public Health; Public Policy Analysis, College of Urban Planning \& Public Affairs, University of Illinois at Chicago
AMG und des GKV - WSG prinzipiell möglich und auch in Bezug auf die industrielle Verblisterung vom Gesetzgeber politisch gewollt. Die Tür zu einer auf den tatsächlichen Bedarf des einzelnen Patienten zugeschnittenen Arzneimittelversorgung ist damit geöffnet.

Nachdem die Verblisterung von Arzneimitteln im stationären Sektor bereits vereinzelt praktiziert wird, richtet sich der Fokus in der gegenwärtigen Diskussion zunehmend auf den ambulanten Sektor und dabei vor allem auf den Wochenblister als einer von mehreren möglichen PAV - Verpackungsformen (Schlauchbeutel, einweg- und wieder verwendbare Kassetten, Tablettenkarusselle und andere).

Mit dem Wochenblister ist der Anspruch verbunden, jeweils über den Zeitraum einer Woche patientenindividuell die Einnahme verschiedener Arzneimittel zu verschiedenen Einnahmezeitpunkten technisch zu unterstützen 
und zu koordinieren. Das Potenzial entsprechend konzipierter Wochenblister wird in der gegenwärtigen Diskussion vorwiegend in einer Aufwandsreduzierung für den Patienten, einer erhöhten Compliance und einem reduzierten Arzneimittelverwurf gesehen. ${ }^{2}$ Als Zielgruppe und mögliche Anwender werden vor allem Chroniker betrachtet.

Nachdem Konzepte für manuell und mit Hilfe von Schlauchbeutelautomaten befüllte Wochenblister für das Apothekenumfeld entwickelt wurden, treten inzwischen erste Anbieter mit industriellen Verblisterungskonzepten in die Öffentlichkeit und führen in Deutschland Pilotprojekte durch. Traditionell an der Arzneimittelversorgung beteiligte Akteure wie Hersteller, Großhändler, Apotheken und Ärzte melden sich teilweise mit befürwortenden (z.B. der Hausärzteverband) und teilweise mit kritischen Kommentaren (z.B. der Verband der Forschenden Arzneimittelhersteller) zu Wort. In der deutschsprachigen Fachwelt werden erste, sich teilweise widersprechende, Gutachten erstellt. Wissenschaftlich belastbare Ergebnisse aus Deutschland liegen noch nicht vor. Eine erste medizinische Publikation aus den USA erschien $2006 .^{3}$

Vor diesem Hintergrund haben sich die Autoren unter anderem im Rahmen des integrierten Vollversorgungsmodells „Gesundes Kinzigtal“ intensiv mit dem möglichen Nutzenpotenzial von Wochenblistern beschäftigt und dabei erstmalig primär die Perspektive der Kostenträger (im Folgenden definiert als Krankenkassen sowie budgetverantwortende Systemanbieter und Managementgesellschaften in Neuen Versorgungsformen) eingenommen. Ausgangspunkt war dabei das Bestreben, die Arzneimittelversorgung chronisch Kranker zu analysieren und ggf. zu optimieren.
Der akute Handlungsbedarf für eine solche Analyse resultiert vor allem aus dem ab 2009 gültigen Gesundheitfonds einschließlich des an Morbidität orientierten Risikostrukturausgleichs der Krankenkassen.

Im Mittelpunkt der Untersuchung standen deshalb die folgenden Fragen: Wie viele Chroniker gibt es und wie viele bzw. welche Arzneimittel konsumieren sie? Kann der Wochenblister einen Beitrag zur Optimierung der Arzneimittelversorgung leisten und wenn ja, welchen? Wie muss ein Wochenblisterkonzept technisch, inhaltlich und organisatorisch ausgestaltet sein? Wie kann es in die bestehenden Funktionsmechanismen des deutschen Gesundheitssystems eingebunden werden?

Durchgeführt wurden eine auf GKV - Echtdaten (ca. 30.000 Versicherte) beruhende Potenzialanalyse sowie einzelne Arzt- und Patientenbefragungen. Dabei wurden Ergebnisse erzielt, die weit über den bisher veröffentlichten Diskussionsstand hinausgehen. Im Rahmen dieser Ausarbeitung werden die Ergebnisse veröffentlicht, die zur Beurteilung der benannten Fragen relevant sind. Damit soll ein Beitrag geleistet werden, um das „echte“ Potenzial von Wochenblistern und konkrete Realisierungsansätze aufzuzeigen.

\section{Datenbasis und Datenanalyse}

Grundlage der Analyse sind die Routinedaten von 29.905 Versicherten der AOK Baden-Württemberg im Kinzigtal aus dem Jahr 2005. Neben den Stammdaten der Versicherten fußt die Datengrundlage auf den für die Ab-

\section{Grafik 1: Versichertenstruktur Modellregion Gesundes Kinzigtal versus GKV nach Alter und Geschlecht}

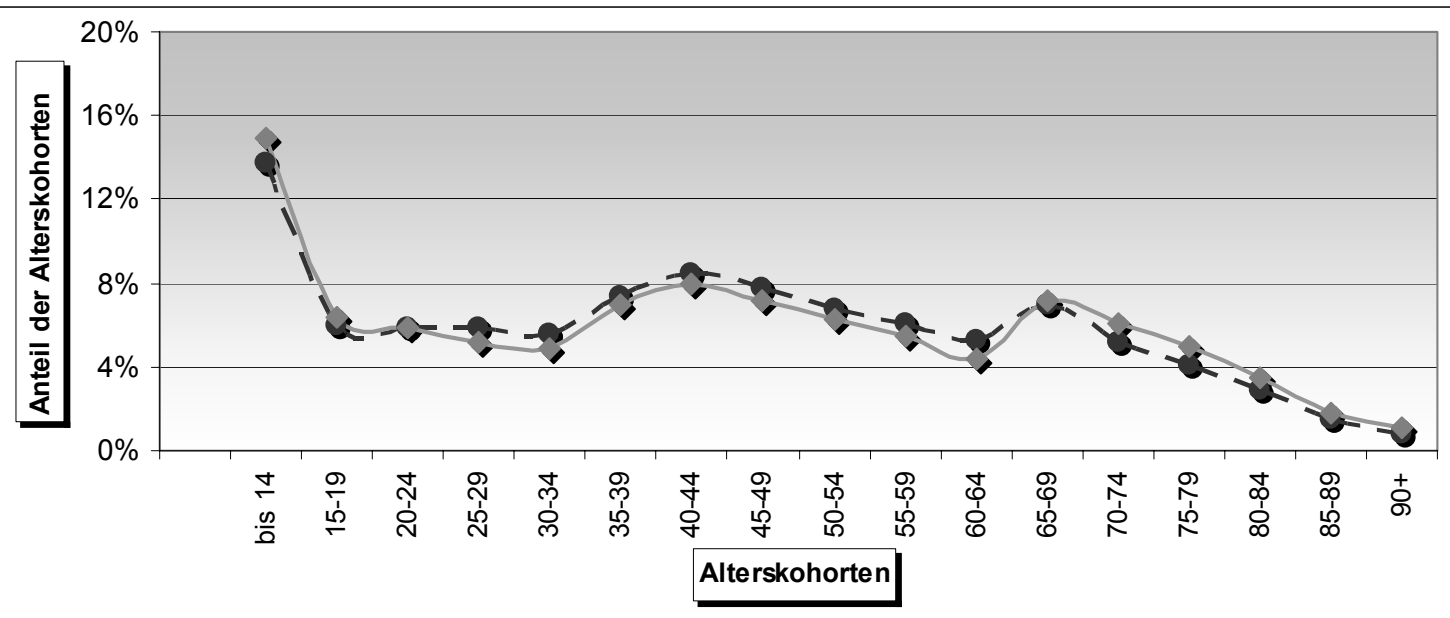

- - Anteil der Alterskohorte an GKV-Gesamtpopulation

$\longrightarrow$ Anteil der Alterskohorte an der Bevölkerung der Modellregion

Quelle: Eigene Erhebungen auf Grundlage der Abrechnungsdaten der Versichertenpopulation in der Modellregion Gesundes Kinzigtal; statistisches Bundesamt 


\section{THEMA}

Grafik 2: Altersspezifischer Anteil der identifizierten Chroniker an der Versichertenpopulation in der Modellregion Gesundes Kinzigtal

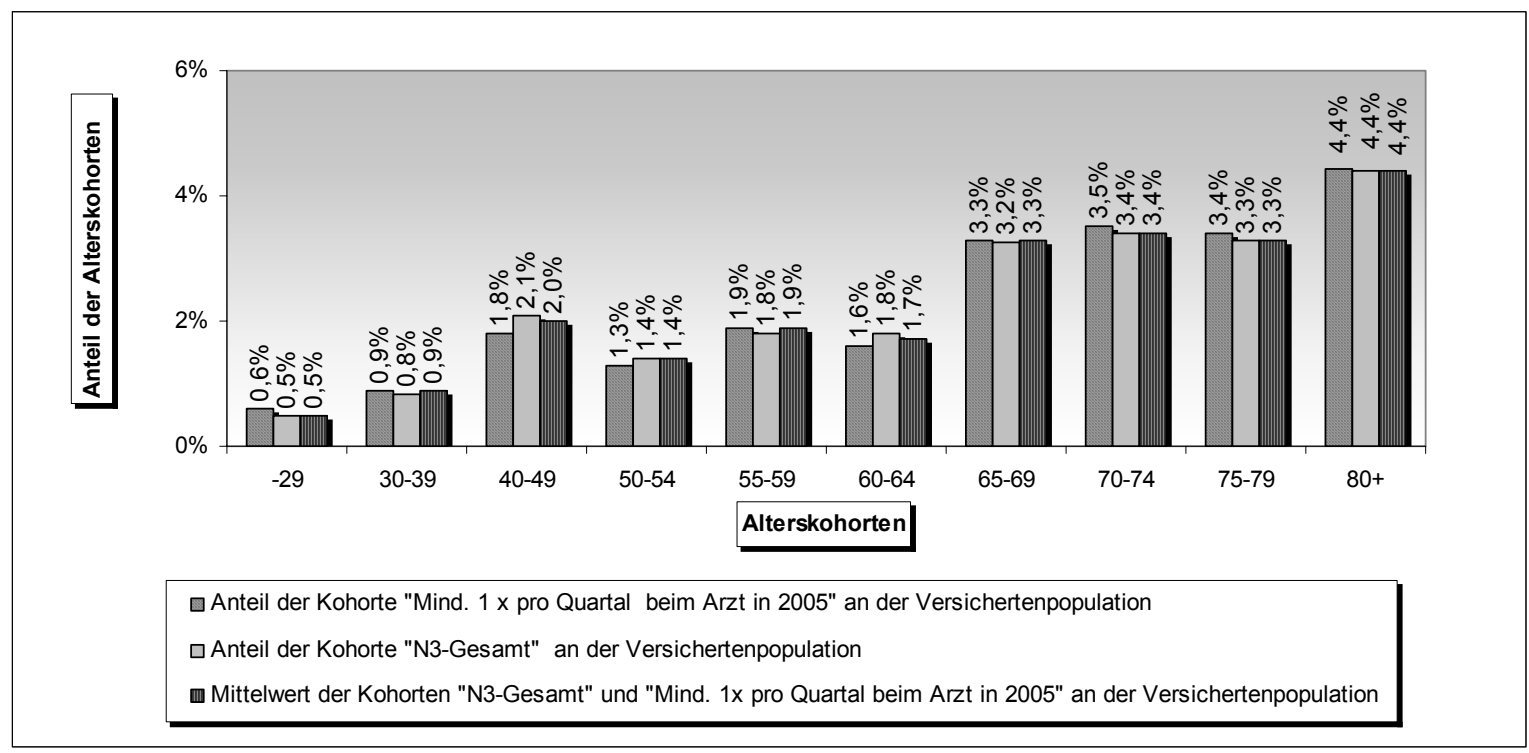

$n=29.905$ Versicherte

Quelle: Eigene Erhebungen auf Grundlage der Abrechnungsdaten der Versichertenpopulation in der Modellregion Gesundes Kinzigtal

Grafik 3: Anteil der einzelnen Alterskohorten an der Versichertenpopulation und den identifizierten Arzneimittelchronikern

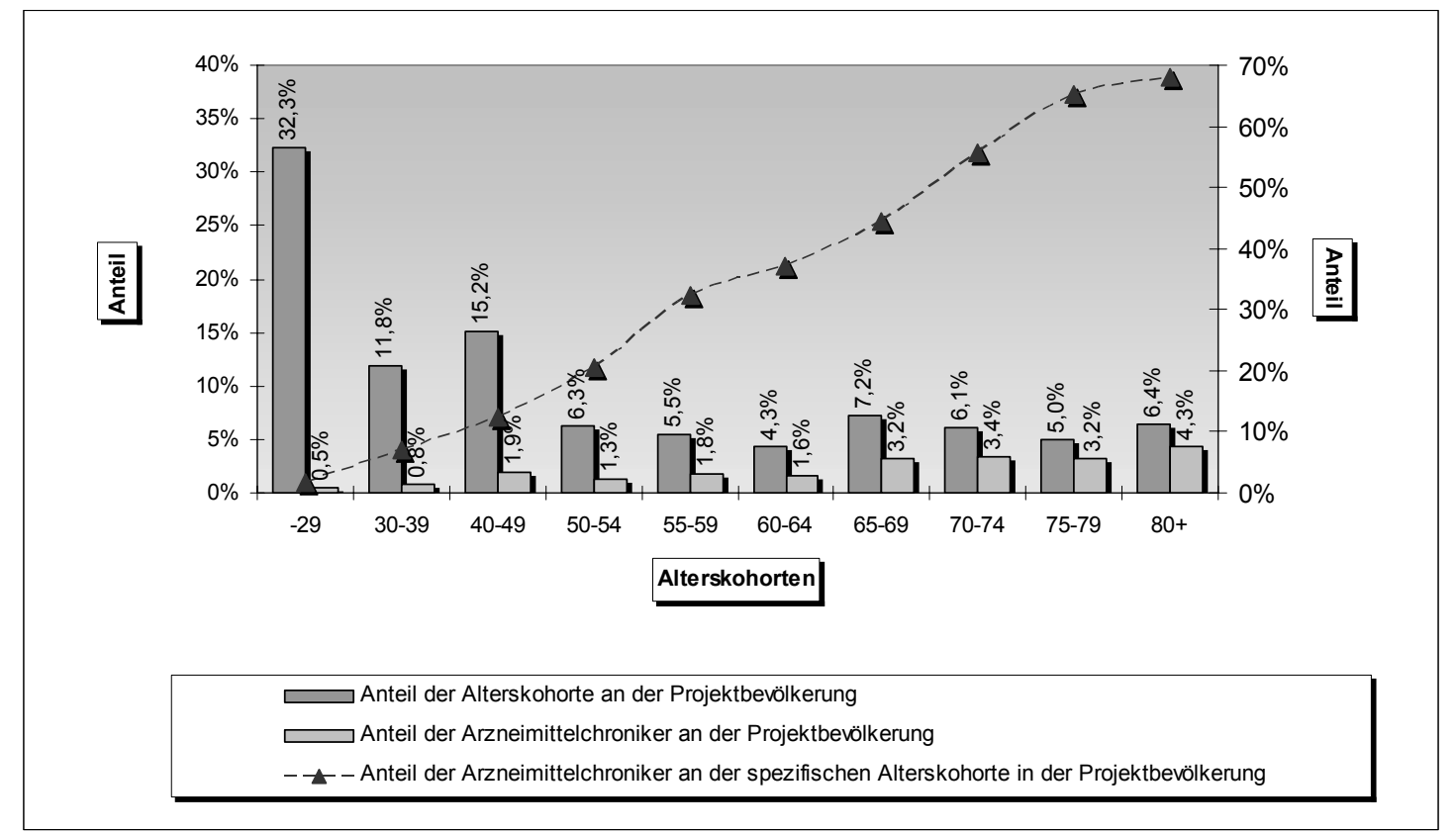

$n$ Versichertenpopulation absolut $\Sigma=100 \%$ bzw. 29.905 Versicherte

Quelle: Eigene Erhebungen auf Grundlage der Abrechnungsdaten der Versichertenpopulation in der Modellregion Gesundes Kinzigtal 


\section{Grafik 4: Kumulierter Anteil der einzelnen Alterskohorten an der Versichertenpopulation und den Arzneimittelchronikern}

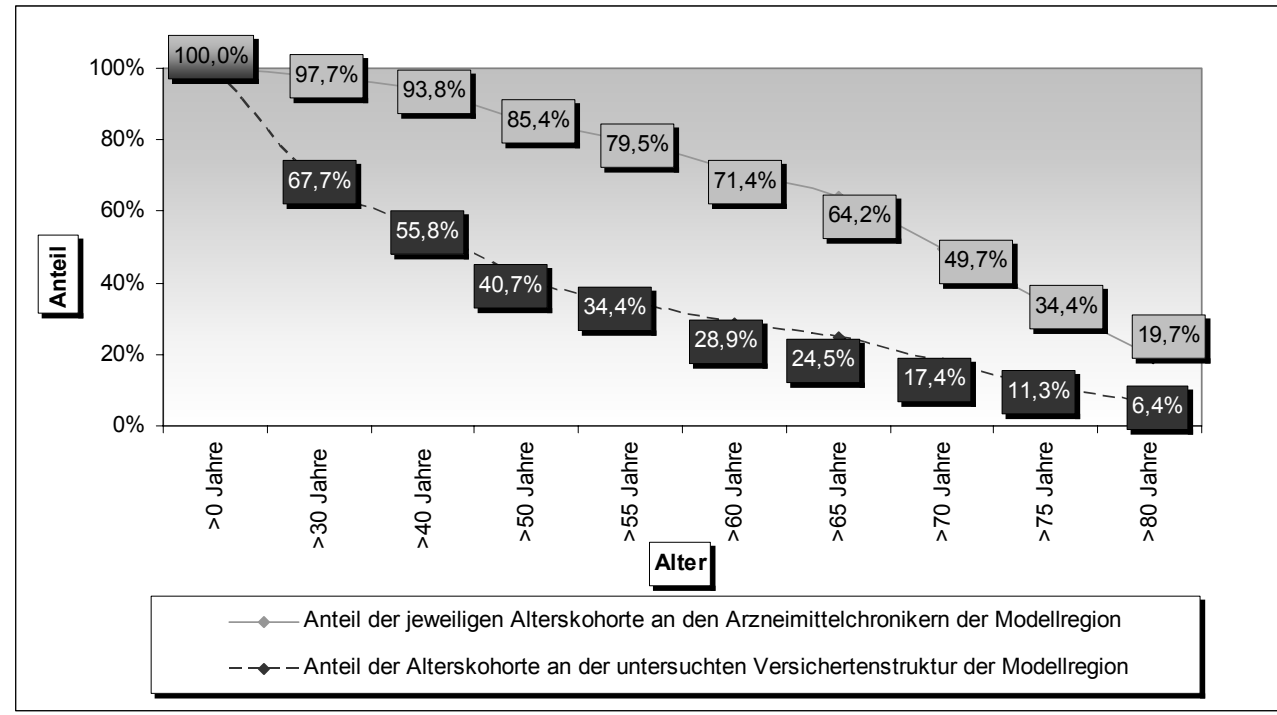

$n$ Arzneimittelchroniker $=6.842$ Versicherte $; n$ Versichertenpopulation $=29.905$ Versicherte

Quelle: Eigene Erhebungen auf Grundlage der Abrechnungsdaten der Versichertenpopulation in der Modellregion Gesundes Kinzigtal

rechnung von Leistungserbringern bei der Krankenkasse eingereichten Leistungsnachweisen. Die von der AOK Baden-Württemberg pseudonymisierten Kosten- und Leistungsdaten wurden anonymisiert aufbereitet.

Ein Vergleich der Versichertenstruktur von Modellregion und GKV ergibt, dass die Versichertenstruktur in der Modellregion in Bezug auf die Parameter Alter und Geschlecht in hohem Maße mit der bundesweiten GKVVersichertenpopulation deskriptiv vergleichbar ist, auch wenn die älteren Jahrgänge leicht überrepräsentiert sind (siehe Grafik 1).

Zur Identifizierung der regelmäßig Arzneimittel konsumierenden Chroniker wurden als Aufgreifkriterien die Regelmäßigkeit von N3-Verordnungen und von Arztkontakten verwendet. Dabei wurden die beiden Aufgreifkriterien wie folgt definiert (siehe Grafik 2):

- Aufgreifkriterium N3: Versicherte, die im ersten Quartal 2005 eine N3 - Verordnung und anschließend entweder im zweiten, dritten oder vierten Quartal mindestens eine weitere N3 - Verordnung aus derselben ATC - Hauptgruppe erhalten haben.

- Aufgreifkriterium Arztkontakte: Versicherte mit mindestens einem Arztbesuch je Quartal und damit mindestens vier Arztkontakten in 2005.

Beide zur Identifizierung der Chroniker definierten Kriterien „Verordnungen N3 “ und „regelmäßiger Arztkontakt“ führen in allen Alterskohorten zu ähnlichen Chronikerprävalenzen. Zur Veranschaulichung wird der entsprechende Mittelwert gebildet und je Alterskohorte in der rechten
Säule (siehe Grafik 2) dargestellt. Für die Versicherten in der Modellregion ergibt sich auf Basis des „Aufgreifkriteriums N3 “ eine Prävalenz von 6.842 Patienten mit regelmäßigem Arzneimittelkonsum. Im Folgenden werden diese Patienten wegen ihres konstanten Arzneimittelkonsums als „Arzneimittelchroniker“ bezeichnet. Ihre Menge entspricht einem relativen Anteil von 22,8\% an der Grundgesamtheit der Versicherten in der Modellregion.

Wenig überraschend steigt der Anteil der jeweiligen Alterskohorte an den Arzneimittelchronikern mit zunehmendem Alter an. Dies gilt insbesondere ab dem 65 Lebensjahr (siehe Grafik 6).

Der Großteil der identifizierten Arzneimittelchroniker ist in den höheren Altersstufen zu finden. 71,4\% sind älter als 60 Jahre; die über 70 Jahre alten Versicherten stellen mit 49,7\% immerhin fast die Hälfte aller Arzneimittelchroniker (siehe Grafik 4). Dass in den jüngeren Alterskohorten eine nicht geringe Anzahl an Arzneimittelchronikern zu finden ist, liegt unter anderem daran, dass z.B. auch alle regelmäßig eingenommenen Kontrazeptiva berücksichtigt sind.

Diese Ergebnisse decken sich in etwa mit einer Studie von Kuhlmey et al. aus dem Jahr 2005, in der 73.193 Versichertendaten der über 60-jährigen Versicherten einer gesetzlichen Krankenkasse analysiert wurden. ${ }^{4}$ Im Rahmen dieser Studie wurde permanenter Arzneimittelkonsum als eines von fünf alternativen Kriterien zur Identifizierung von Chronikern definiert als: „In drei von sechs Quartalen mussten zusammenhängende oder nicht zusammenhängende Arzneimittelverordnungen aus derselben ATC - 
Grafik 5: Verordnungshäufigkeit für die identifizierten Arzneimittelchroniker pro Jahr nach Alterskohorten

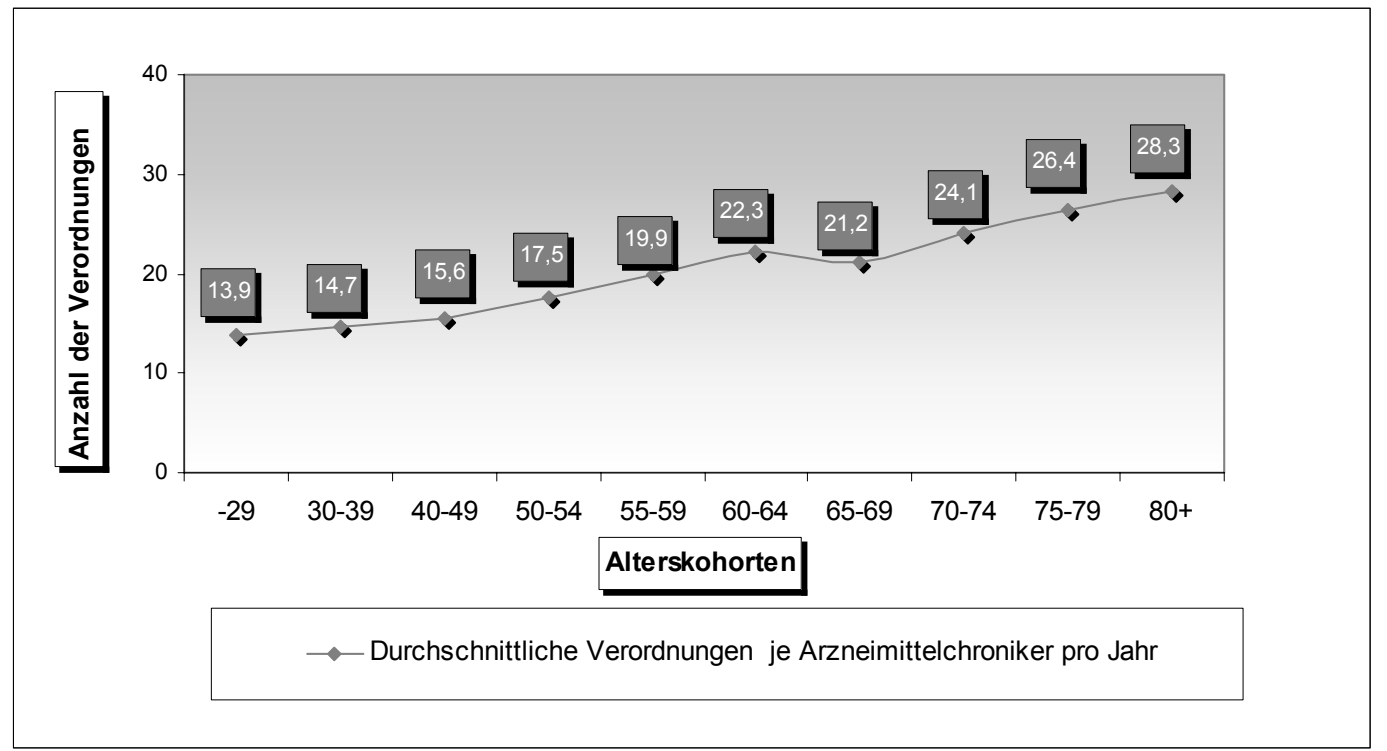

Quelle: Eigene Erhebungen auf Grundlage der Abrechnungsdaten der Versichertenpopulation in der Modellregion Gesundes Kinzigtal

Hauptgruppe vorliegen“. Dabei wurde herausgefunden, dass 73\% der über 60-jährigen Versicherten regelmäßig mindestens ein Medikament erhalten, das nachweislich der Behandlung einer chronischen Krankheit dient.

\subsection{Herleitung und Prognose der Anzahl} "Arzneimittelchroniker" für Gesamtdeutschland

Um die Relevanz von Wochenblistern für das Gesundheitssystem, die Kostenträger und die Patienten beurteilen zu können, werden nachfolgend die in der eigenen Erhebung ermittelten alters- und geschlechtsspezifischen Prävalenzen der Arzneimittelchroniker auf die gegenwärtige Bevölkerungsstruktur in Gesamtdeutschland übertragen.

Von den ca. 82,5 Millionen in Deutschland lebenden Menschen wären unter der Annahme einer weitgehend repräsentativen Versichertenpopulation in der Modellregion Gesundes Kinzigtal 22,8\% und damit ca. 18,8 Mio. Menschen chronisch krank in dem Sinne, dass sie dauerhaft mindestens ein Arzneimittel konsumieren („Arzneimittelchroniker“). Davon wären nahezu 15,2 Mio.

Menschen älter als 60 Jahre. Diese an sich schon beeindruckende Größenordnung gewinnt dadurch noch an Bedeutung, dass die Bevölkerung in Deutschland zunehmend altert. Prognostiziert wird ein Alterungsprozess, der vor allem von den vier folgenden Ausprägungen gekennzeichnet sein wird: Erstens von einer Verlängerung der Lebenszeit, zweitens von einer absolut steigenden Menge alter Menschen, drittens von einem steigenden Altersquotienten und viertens von einer überproportionalen Zunahme Hochbetagter (über 85-Jährige). Unter Fortschreibung des in der Untersuchung ermittelten Anteils der Arzneimittelchroniker an der jeweiligen Alterskohorte bedeutet dies bei Bezugnahme auf die prognostizierte Bevölkerungsstruktur für Gesamtdeutschland z.B. für das Jahr 2020 eine Zunahme der Arzneimittelchroniker um weitere ca. 3 Millionen auf dann ca. 21,8 Millionen Menschen, auch wenn die Gesamtbevölkerung entsprechend der Prognosen des Statistischen Bundesamtes in diesem Zeitraum um ca. 2,1 Millionen Menschen abnehmen wird. Dieser Prognose liegen die vom Statistischen Bundesamt dokumentierten Demografieprognosen zugrunde. ${ }^{5}$ Hinzu kommt, dass durch die absehbar steigende Lebenserwartung die Manifestation und Chronifizierung vormals latent vorhandener Krankheiten zunehmen und sich die Dauer chronischer Krankheiten und damit auch der damit verbundene Arzneimittelkonsum voraussichtlich erhöhen wird.

\subsection{Verordnungen der "Arzneimittelchroniker"}

Nachdem die Arzneimittelchroniker in ihrer Menge und Altersstruktur erfasst wurden, stellt sich die Frage, wie viele und welche Verordnungen sie erhalten.

Die durchschnittliche Anzahl der Verordnungen steigt mit zunehmendem Alter an. Bei den über 60-Jährigen beträgt die durchschnittliche Anzahl der Verordnungen in Abhängigkeit von der konkreten Altersstufe zwischen 21,2 und 28,3 p.a. (siehe Grafik 5). Um die Kontinuität der Verordnungen im Jahresverlauf zu überprüfen, wurde nachfolgend die absolute Verordnungshäufigkeit in den betreffenden vier Quartalen 2005 gegenüber gestellt. In der Analyse zeigte sich, dass ca. $91 \%$ der Verordnungsmenge in 
jedem der vier beschriebenen Quartale übereinstimmen. Die Verordnungsmenge ist also offensichtlich weitgehend unabhängig von einzelnen Quartalen und damit weitgehend konstant.

Anschließend wurde untersucht, wie viele Arzneimittelchroniker dauerhaft welche Mindestanzahl an Wirkstoffen verordnet bekommen. Das dabei untersuchte Kontinuum reicht von minimal einem bis zu minimal zehn Wirkstoffen je Patient und Quartal. Diese Zuordnung schließt aus, dass in einzelnen Quartalen weniger Arzneimittel verordnet wurden. Sie schließt nicht aus, dass in einzelnen Quartalen auch teilweise mehr Arzneimittel verordnet wurden (siehe Tabelle 1). vor allem Liquida (inkl. Insulin), Topica, Inhalate und Teststäbchen z.B. bei Diabetes. Deren spezifischer Anteil hätte nur mit großem Aufwand aus den Routinedaten ermittelt werden können, darauf wurde hier zunächst verzichtet. Wille \& Wolff gehen in einer eigenen Veröffentlichung von einem Anteil von ca. 20\% nicht verblisterbarer Arzneimittel aus. ${ }^{6}$ Bei einer entsprechenden Verringerung der obigen Zahlen würden daraus ca. 6,4. Mio. Arzneimittelchroniker (mit mindestens drei konstant verordneten oral einzunehmenden Solida-Wirkstoffen) als realistische Wochenblisterzielgruppe resultieren. Bei der relevanten Zielgruppe handelt es sich somit zu einem erheblichen Anteil um multimorbide

Tabelle 1: $\quad$ Arzneimittelchroniker mit Zuordnung zu den konstanten Wirkstoffmengen je Quartal

\begin{tabular}{|c|c|c|c|c|c|c|c|c|c|c|c|}
\hline $\begin{array}{l}\text { Alter der } \\
\text { Arzneimittel- } \\
\text { chroniker }\end{array}$ & $\begin{array}{c}\text { Mind. } 1 \\
\text { Wirkstoff }\end{array}$ & $\begin{array}{c}\text { Mind. } 2 \\
\text { Wirkstoffe }\end{array}$ & $\begin{array}{c}\text { Mind. } 3 \\
\text { Wirkstoffe }\end{array}$ & $\begin{array}{c}\text { Mind. } 4 \\
\text { Wirkstoffe }\end{array}$ & $\begin{array}{c}\text { Mind. } 5 \\
\text { Wirkstoffe }\end{array}$ & $\begin{array}{c}\text { Mind. } 6 \\
\text { Wirkstoffe }\end{array}$ & $\begin{array}{c}\text { Mind. } 7 \\
\text { Wirkstoffe }\end{array}$ & $\begin{array}{c}\text { Mind. } 8 \\
\text { Wirkstoffe }\end{array}$ & $\begin{array}{c}\text { Mind. } 9 \\
\text { Wirkstoffe }\end{array}$ & $\begin{array}{c}\text { Mind. } 10 \\
\text { Wirkstoffe }\end{array}$ & Summe \\
\hline-59 & 1.884 .280 & 772.440 & 428.428 & 204.976 & 121.024 & 68.586 & 38.691 & 25.120 & 16.236 & 29.714 & 3.589 .445 \\
\hline $60+$ & 4.917 .080 & 3.327 .560 & 2.431 .572 & 1.635 .024 & 1.118 .976 & 641.485 & 452.358 & 288.880 & 181.705 & 217.237 & 15.211 .876 \\
\hline $\begin{array}{l}\text { relat. Anteil } \\
60+\end{array}$ & $72 \%$ & $81 \%$ & $85 \%$ & $89 \%$ & $90 \%$ & $90 \%$ & $92 \%$ & $92 \%$ & $92 \%$ & $88 \%$ & $81 \%$ \\
\hline $\begin{array}{l}\text { Durch- } \\
\text { schnittsalter } \\
\text { in Jahren }\end{array}$ & 66,1 & 70,0 & 71,8 & 73,1 & 73,8 & 74,5 & 74,5 & 74,6 & 73,3 & 73,1 & 69,9 \\
\hline Summe & 6.800 .000 & 4.100 .000 & 2.860 .000 & 1.840 .000 & 1.240 .000 & 710.000 & 490.000 & 314.000 & 198.000 & 247.000 & 18.799 .000 \\
\hline
\end{tabular}

Quelle: Ermittlung der Werte als Hochrechnung der Erhebungsergebnisse/Abrechnungsdaten der Versichertenpopulation in der Modellregion auf die Gesamtbevölkerung Deutschlands

Bei Übertragung der altersspezifischen Arzneimittelchronikerprävalenz aus dem Modellprojekt auf die Gesamtbevölkerung bekommen deutschlandweit ca. 18,8 Mio. Menschen konstant mindestens ein Arzneimittel verordnet. Davon erhalten ca. 12 Mio. Menschen (Reduzierung der Gesamtsumme um die Patienten, die konstant nur einen Wirkstoff erhalten) konstant mindestens zwei Wirkstoffe und ca. 8 Mio. Menschen (Reduzierung der Gesamtsumme um die Patienten, die konstant nur einen bzw. zwei Wirkstoffe erhalten) konstant mindestens drei Wirkstoffe.

Zur Ermittlung der realistischen Zielgruppe ist diese Patientenanzahl um die Patienten zu reduzieren, deren Medikation nicht wochenblistergeeignet ist. Dies betrifft
Chroniker. Es ist zusätzlich zu berücksichtigen, dass auch ein relevanter Anteil der Arzneimittelchroniker mit konstant mindestens zwei verordneten oral einzunehmenden Solida-Wirkstoffen prinzipiell „wochenblistergeeignet“ ist. Aus Gründen der abgesicherten Vorgehensweise wird aber darauf verzichtet, diese Patientenklientel als Bestandteil der realistischen Zielgruppe zu berücksichtigen.

Nachdem die Anzahl konstanter Verordnungen je Arzneimittelchroniker und Alterskohorte ermittelt wurde, wurde in einem nächsten Schritt untersucht, wie viele Ärzte jeweils an den Verordnungen für den einzelnen Arzneimittelchroniker beteiligt sind. Dabei wurden alle Arzneimittelchroniker unabhängig von der Anzahl der 
Grafik 6: Arzneimittelkosten der Arzneimittelchroniker je Quartal

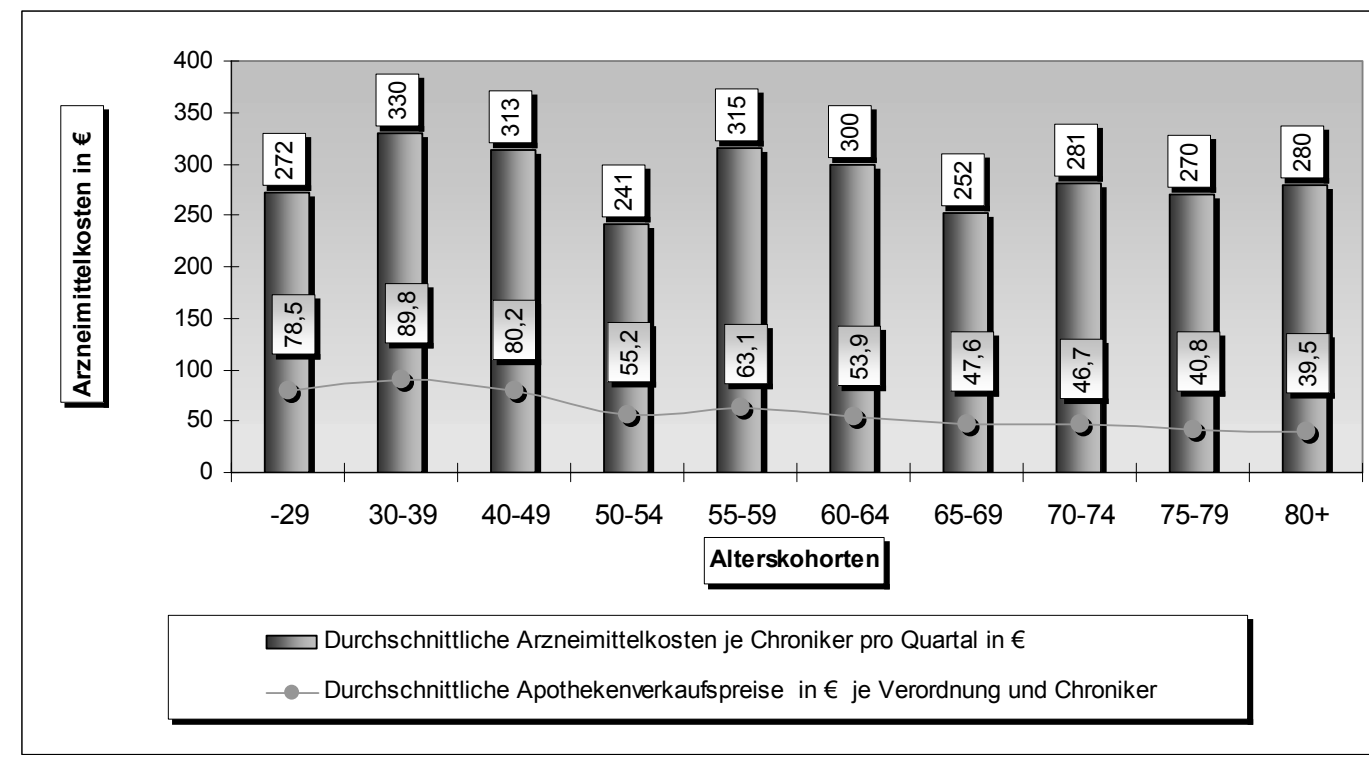

$n$ Chroniker $=6.842$ Versicherte

Quelle: Eigene Erhebungen auf Grundlage der Abrechnungsdaten der Versichertenpopulation in der Modellregion Gesundes Kinzigtal

konstanten Verordnungen berücksichtigt. Die Anzahl der Verordnungen je Arzt und Patient beträgt in der Zielgruppe 60plus zwischen 10 und 14 p.a.; sie steigt mit zunehmenden Alter der Patienten erwartungsgemäß an. Die Anzahl der an der Medikation des einzelnen Patienten durchschnittlich beteiligten Ärzte hingegen nimmt mit zunehmendem Alter leicht ab. Sie beträgt bei den über 60-Jährigen etwas mehr als zwei. Dies sind in aller Regel Hausarzt und Internist.

In einem nächsten Schritt kam es darauf an, die mit den identifizierten Dauerverordnungen verbundenen Arzneimitteltherapiekosten für die identifizierte Zielgruppe zu erfassen.

Die durchschnittlichen Arzneimittelkosten je Arzneimittelchroniker betragen (unabhängig von der Anzahl der konstant verordneten Arzneimittel je Arzneimittelchroniker) in Abhängigkeit vom Alter etwa zwischen ca. 1.000 $€$ und ca. $1.300 €$ p.a. (siehe Grafik 6). Der Durchschnittspreis je Verordnung (Apothekenverkaufspreis) nimmt mit zunehmendem Alter deutlich ab; er liegt bei den über $60-J a ̈ h r i g e n ~ b e i ~ 46,80 €$. Während also die Anzahl der Verordnungen mit zunehmendem Alter zunimmt, zeigen sich die damit verbundenen Arzneimittelkosten in der Jahresbetrachtung deutlich unabhängiger vom Alter der Patienten. Dies beruht offensichtlich unter anderem auf altersspezifischen Krankheitsbildern und dem in den höheren Altersklassen deutlich höheren Generikaanteil.

Welche mit regelmäßigem Arzneimittelkonsum verbundenen Krankheitsbilder sind relevant? Zur ersten Abgren- zung wird Bezug auf das anatomisch - therapeutisch - chemische Klassifikationssystem (ATC) und dessen Methodik der Substanzenzuordnung genommen.

Innerhalb der Hauptgruppen der ersten ATC - Gliederungsebene (Organ- bzw. Systembezug) überwiegen Substanzen, die zur Behandlung von Krankheiten des kardiovaskulären Systems eingesetzt werden. Auch Substanzen, die mit der Behandlung von Krankheiten des Zentralnervensystems, des Verdauungstraktes und Stoffwechsels sowie des Muskel- und Skelettsystems in Verbindung stehen, besitzen eine hohe Relevanz. Für die identifizierten Arzneimittelchroniker stellen die vier relevantesten ATC - Codes der ersten Ebene einen Anteil von ca. 65\% an allen verordneten Substanzen dar (siehe Grafik 7).

Die Analyse der zweiten ATC-Ebene ergab, dass bei den Verordnungen Substanzen mit den folgenden Therapiebezügen überwiegen: Psychoanaleptika, Antidiabetica, Antirheumatica, Betarezeptorenblocker, Analgetica, systematische Antibiotika, Schilddrüsentherapie, Antacida, Asthmapräparate und Diuretica (alle mit mehr als 3\%). Diese 12 auf Therapiegruppen bezogenen Substanzen machen einen Anteil von ca. 50\% an allen Verordnungen bei den Arzneimittelchronikern aus.

Ähnliche Zuordnungen wurden in verschiedenen Veröffentlichungen, darunter im Krankenhausreport $1999^{7}$ und einer Analyse von Glaeske ${ }^{8}$ vorgenommen. Die mit permanentem Arzneimittelkonsum verbundene Morbidität und die dafür verwendeten Substanzen sind in ihren Grundzügen folglich klar umrissen. 
Grafik 7: Relativer Anteil der einzelnen ATC - Codes an allen ATC - Codes der 1. Ebene für die Zielgruppe der identifizierten Arzneimittelchroniker

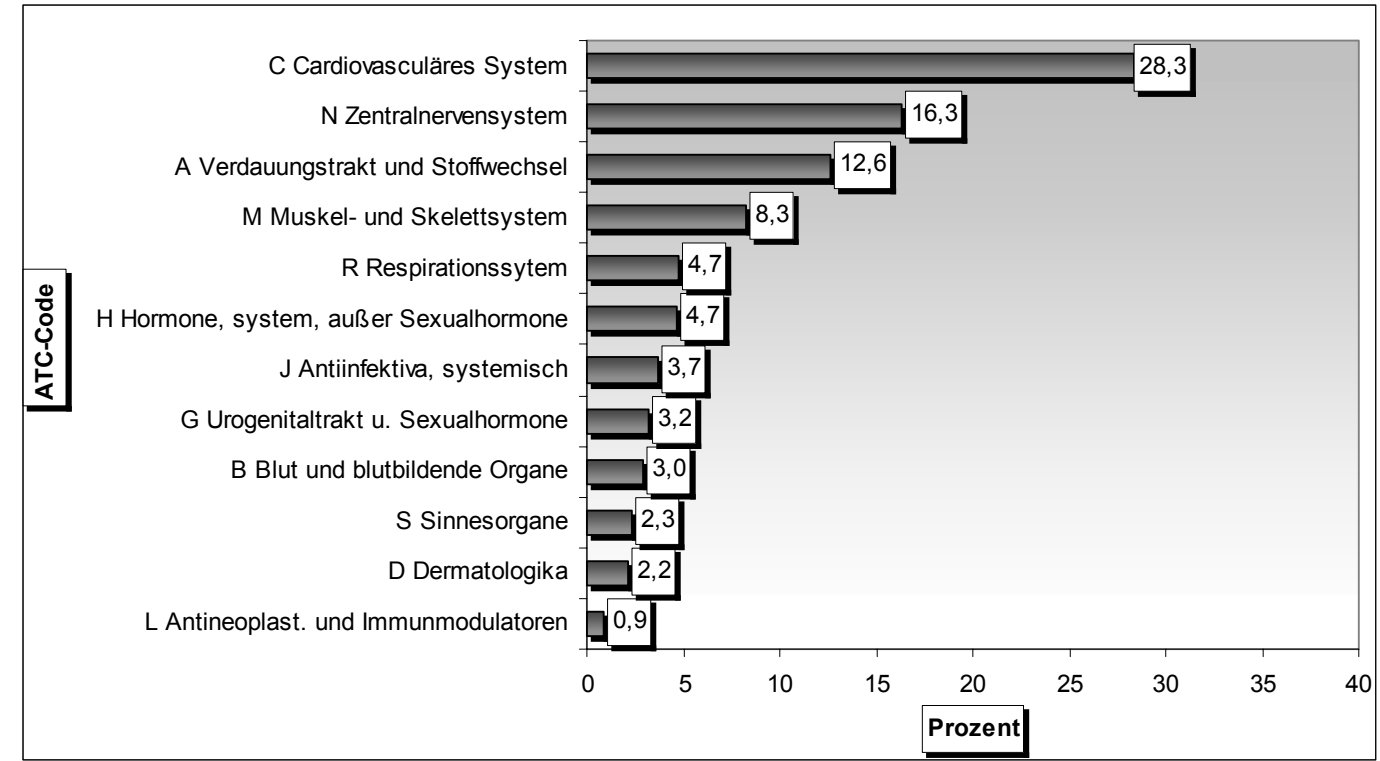

Quelle: Eigene Erhebungen auf Grundlage der Abrechnungsdaten der Versichertenpopulation in der Modellregion Gesundes Kinzigtal

\subsection{Multimorbide Chroniker als Zielgruppe}

Als Ergebnis der durchgeführten Erhebung wird zusammengefasst: Die für den Wochenblister relevante Zielgruppe von Patienten mit dauerhaft mindestens drei zeitgleich eingenommenen Solida-Wirkstoffen ist mit ca. 6,4 Millionen Menschen deutlich größer als erwartet und wird demografiebedingt noch deutlich an Relevanz zunehmen. Sie besteht vor allem aus den über 60-jährigen chronisch kranken Patienten mit einer sich aus verschiedenen Arzneimitteln zusammensetzenden und täglich einzunehmenden Gesamtmedikation. Zielgruppe von Wochenblistern sind damit ältere, chronisch kranke und multimorbide Patienten.

Chronische Krankheiten und Multimorbidität sind aber traditionell nicht im Fokus des deutschen Gesundheitssystems; belastbare Aussagen über den Arzneimittelkonsum chronisch kranker Patienten in Deutschland liegen nur vereinzelt vor. Viele Aspekte in der Versorgung chronischer Krankheiten sind auch deshalb noch unerforscht, weil die Anforderungen an die Versorgung von Chronikern sehr komplex sind und neben der Arzneimitteltherapie beispielsweise auch Elemente von Gesundheitsförderung, Prävention, Kuration, Rehabilitation und Pflege koordiniert werden müssen. Hinzu kommt, dass die Versorgung von Chronikern vor allem die Vernetzung der Leistungserbringer und die Berücksichtigung von Umfeld und Lebenssituation der Patienten erfordert. Beide Aspekte sind im deutschen Gesundheitssystem noch wenig ausgeprägt. Folge davon ist eine vom Sachverständigenrat zur Begutachtung der Entwicklung im Gesundheitswesen (SVR, ehemals: Sachverständigenrat für die Konzertierte Aktion im Gesundheitswesen) 2000/2001 festgestellte Ressourcenverschwendung bei der Versorgung chronischer Patienten sowohl in Form von Unter- als auch Über- und Fehlversorgung ${ }^{9}$. Dies gilt auch für die Arzneimitteltherapie als einem Eckpfeiler bei der Therapie chronischer Krankheiten und vor allem bei den Patienten, die an multiplen Gesundheitsbeeinträchtigungen leiden. ${ }^{10}$

Die verbesserte Versorgung chronisch kranker und oftmals multimorbider älterer Patienten ist damit von großer gesellschaftlicher und ökonomischer Relevanz. Der Gesetzgeber hat dies erkannt. Er entwickelt zunehmend regulatorische Elemente, die auf eine Versorgungsverbesserung für eben diese Zielgruppe abzielen. Beispielhaft seien genannt der eingeforderte Bezug der Integrierten Versorgung zu Volkskrankheiten, die strukturierten Behandlungsprogramme für bestimmte chronische Krankheiten (DMP), obligatorische Hausarztverträge aller gesetzlichen Krankenkassen und vor allem der ab 2009 geplante morbiditätsorientierte Risikostrukturausgleich im Gesundheitsfonds.

\section{Stand der Wissenschaft}

Auch wenn international bereits einige Erfahrungen mit der individuellen Neuverblisterung von Arzneimitteln gemacht wurden (u.a. in Skandinavien, vor allem in Schweden, Australien, den USA, Kanada, Großbritannien und den Niederlanden), wird sie bislang in keinem Land flächendeckend praktiziert. ${ }^{11}$ Die patientenindividuelle Verblisterung erfolgt in den meisten Ländern manuell oder mit Hilfe von Automaten (ausgelegt auf eine stark 


\section{THEMA}

begrenzte Anzahl an Patienten) und wird oft im MultidoseSystem in Krankenhäusern, Alten- und Pflegeheimen oder der häuslichen Betreuung verwendet. In allen relevanten Ländern bleibt die Verblisterungstechnik weit hinter den gegenwärtig in Deutschland diskutierten industriellen Möglichkeiten zurück. Kosten-Nutzen-Analysen zum Einsatz der patientenindividuell neu verblisterten Arzneimittel liegen in keinem der genannten Länder vor. ${ }^{12}$

Die Motivation zur Verwendung von Blisterverpackungen resultiert international vorwiegend aus dem Bestreben, die Arzneimittelsicherheit zu erhöhen. So wird die Einführung von Wochenblistern in den USA vor allem unter dem Aspekt der zwar kostengünstigen, unter dem Aspekt der Arzneimittelsicherheit (in Anwendung und Stabilität der Medikamente) aber kritisch zu beurteilenden traditionellen Glas- und Plastikcontainerverpackungen geführt.

Seit 2006 liegt eine erste wissenschaftliche Publikation ${ }^{13}$ zu einem randomisierten und kontrollierten Versuch mit Blisterkarten vor. Hierbei wurde die Compliancesteigerung und Verbesserung der Blutdruck- und Fettwerte von 200 Patienten über einen Zeitraum von 14 Monaten beobachtet. Im Vergleich zur Kontrollgruppe stieg die Compliance von etwa $60 \%$ hoch signifikant $(\mathrm{P}<.001)$ auf weit über $90 \%$. Dies korrelierte mit einer signifikanten $(\mathrm{P}=.02)$ Verbesserung des systolischen Blutdrucks von 133,2 mmHg auf 129,9 mmHg. Die Fettwerte (LDL-C) veränderten sich nicht signifikant. Welche Kostenersparnis diese Ergebnisse für ein Gesundheitssystem mit sich bringen, ist mit dieser Studie noch nicht belegt. Dass aber ein medizinisch hochrelevanter Laborparameter wie der Blutdruck durch die Complianceverbesserung einer Blisterkarte nachgewiesen wurde, ist gesundheitsökonomisch von hoher Relevanz.

In Deutschland wird das Potenzial von PAV und Wochenblistern gegenwärtig primär unter ökonomischen Gesichtspunkten und nur partiell unter dem Aspekt der Arzneimittelsicherheit diskutiert. Der Schwerpunkt der Diskussion liegt hierbei weltweit erstmals auf der industriellen Form der Verblisterung. Im Unterschied zu der umfassenden internationalen Literatur zum Potenzial der Verblisterung generell, hat eine wissenschaftliche Analyse des Potenzials industriell gefertigter Wochenblister weltweit noch nicht stattgefunden. Diese Diskussion beginnt in Deutschland. Aus den Reihen der Wissenschaftler haben sich in Deutschland bis heute vor allem Lauterbach et al. ${ }^{14}$, Düsing ${ }^{15}$ (beide prinzipiell positiv), Wille $\&$ Wolff ${ }^{16}$ (skeptisch) und Glaeske ${ }^{17}$ (unter dem Aspekt der Relevanz einzelner Wirkstoffe) mit der industriellen Verblisterung auseinander gesetzt. Dabei beziehen sich die vorliegenden Ausarbeitungen der benannten Wissenschaftler vorwiegend auf das Konzept eines einzelnen Anbieters.

In einem 2004 für die assist Pharma $\mathrm{GmbH}^{18}$ erstellten Gutachten beschreibt Lauterbach ${ }^{19}$ Pflegeheime und die ambulante häusliche Pflege als Haupteinsatzgebiete für industriell hergestellte Wochenblister. Einen möglichen Vorteil für Krankenkassen sieht er in erster Linie in einer steigenden Compliance, einem reduzierten Arzneimittelverwurf und einem vermehrten Einsatz von Generika.
Wille \& Wolff ${ }^{20}$ setzten sich in ihrer 2006 im Auftrag des Verbandes Forschender Arzneimittelhersteller (VFA) erstellten Ausarbeitung kritisch mit der von Lauterbach et al. in Aussicht gestellten Compliancesteigerung durch Wochenblister auseinander. Diese sehen sie unter dem Hinweis auf die multifaktoriellen Ursachen von Therapietreue nur sehr bedingt. In ihrer Einschätzung kann durch die mit einem Wochenblister verbundene Vereinfachung des Einnahmeschemas lediglich im Bereich der durch die Komplexität der medikamentösen Therapie verursachten Noncompliance eine Compliancesteigerung erreicht werden. Auch einen signifikant reduzierten Arzneimittelverwurf halten sie bei der automatisch mit einem Wochenblister verbundenen Dauermedikation für wenig relevant. Wille $\&$ Wolff sehen für das von Lauterbach et al. beschriebene Konzept weder für das Gesundheitssystem noch für die Kostenträger eine positive Kosten-Nutzen-Relation. Den klar definierten Kosten für Produktion, Herstellung und Vertrieb steht nach ihrer Auffassung ein deutlich geringerer und zudem nicht quantifizierbarer Nutzen gegenüber. ${ }^{21}$

Düsing setzt sich ebenfalls im Auftrag der assist Pharma $\mathrm{GmbH}$ mit dem Potenzial von Wochenblistern auseinander und geht dabei vor allem auf deren Potenzial, die Therapietreue der Patienten zu steigern, ein. Düsing verwendet für Therapietreue den Begriff der Adhärenz und beschreibt, dass Compliance neben Akzeptanz und Persistenz lediglich eines von drei Elementen der Therapietreue darstellt. ${ }^{22}$ Den potenziellen Effekt von Wochenblistern auf die Therapietreue sieht er vor allem darin, durch technische Unterstützung Vergesslichkeit zu reduzieren und Überforderung zu vermeiden. Einen darüber hinaus gehenden Einfluss der Neuverblisterung auf die Adhärenz sieht auch er nicht als erwiesen an und verweist auf einen entsprechenden Forschungsbedarf. Interessant ist, dass Düsing den Zusammenhang von sinkender Therapieadhärenz bei Zunahme der täglich einzunehmenden Medikamentendosen thematisiert und damit den potenziell besonders hohen Nutzen von Wochenblistern für diejenigen Patienten herausstellt, die mehrere Wirkstoffe gleichzeitig einnehmen (oft auch als Polipharmazie beschrieben).

Glaeske ermittelt im Auftrag der Kohl Medical AG den Anteil der Polipharmazie an den Arzneimittelverordnungen einer Krankenkasse (GEK - Routinedaten aus 2005), der sich für die individuelle Verblisterung eignet. Selektiert wurden in dieser Studie ausschließlich Patienten mit mindestens drei Wirkstoffgruppen in fester oraler Darreichungsform. Die verschiedenen Wirkstärken wurden je Wirkstoff auf die jeweils niedrigste reduziert. Das Ergebnis ist eine Liste der 400 am häufigsten von Allgemeinmedizinern und Internisten verordneten Wirkstoffe. ${ }^{23}$ Damit leistet Glaeske auf wissenschaftlicher Ebene einen ersten Beitrag zu möglichen Wirkstoffinhalten eines Wochenblisters, auch wenn die für eine angemessene Konzeption eines Wochenblisters zwingend notwendige Berücksichtigung unterschiedlicher Wirkstärken je Wirkstoff noch nicht berücksichtigt ist.

Es ist ersichtlich geworden, dass die Wissenschaft begonnen hat, sich mit dem Potenzial industriell gefertigter 
Wochenblister auseinander zu setzen. Dabei kommen die einzelnen Autoren zu teilweise erheblich divergierenden Einschätzungen. Die wissenschaftliche Beurteilung verschiedener Aspekte steht genau so wie eine Gesamtbeurteilung von Potenzial und Relevanz der Wochenblister noch aus.
Diese vier Kriteriengruppen sind von entscheidender Bedeutung für die Bewertung von Wochenblisterkonzeptionen. Im Folgenden wird beschrieben, welche konkreten Anforderungen auf dieser Basis an eine Erfolg versprechende Wochenblisterkonzeption zu stellen sind.

Tabelle 2: $\quad$ Kriterien für die Beurteilung von Wochenblistern

\begin{tabular}{|c|c|c|c|}
\hline Kriteriengruppe & Kriterien & \multicolumn{2}{|l|}{ Elemente } \\
\hline Inhalt/Fachlich & $\begin{array}{l}\text { Bedienung der relevanten } \\
\text { Zielgruppe }\end{array}$ & $\begin{array}{l}\text { Ältere multimorbide Chro- } \\
\text { niker mit dauerhaft mehr als } \\
\text { zwei Wirkstoffen in fester } \\
\text { oraler } \\
\text { Darreichungsform }\end{array}$ & $\begin{array}{l}\text { Bedienung des relevanten Krank- } \\
\text { heitsspektrums einzelner multimor- } \\
\text { bider Chroniker und nicht einzelner } \\
\text { Krankheiten }\end{array}$ \\
\hline \multirow[t]{2}{*}{ Technisch } & Arzneimittelsicherheit & $\begin{array}{l}\text { Einhaltung des bei Fertigarz- } \\
\text { neimitteln/ Solida in Deutsch- } \\
\text { land geltenden technischen } \\
\text { Herstellungs- und Verpak- } \\
\text { kungsstandards }\end{array}$ & $\begin{array}{l}\text { Erhöhung der Arzneimittelsicherheit } \\
\text { bei multimorbiden Chronikern durch } \\
\text { Interaktionschecks und Überprüfung } \\
\text { auf Wechselwirkungen (ärztlich } \\
\text { - pharmazeutisch) }\end{array}$ \\
\hline & $\begin{array}{l}\text { Convenience für ältere } \\
\text { und kranke Patienten }\end{array}$ & $\begin{array}{l}\text { Aufwandreduzierung bei } \\
\text { Bezug und Einnahme } \\
\text { (organisatorisch) }\end{array}$ & $\begin{array}{l}\text { Erleichterung bei der Handhabung } \\
\text { (technisch) }\end{array}$ \\
\hline \multirow[t]{2}{*}{ Kaufmännisch } & $\begin{array}{l}\text { Positive Kosten-Nutzen } \\
\text { Relation für die Kosten- } \\
\text { träger }\end{array}$ & $\begin{array}{l}\text { Monetär konkret bewertbare } \\
\text { Effekte }\end{array}$ & $\begin{array}{l}\text { Überlegene Kosteneffektivität im } \\
\text { Vergleich zu Alternativen }\end{array}$ \\
\hline & Marketingnutzen & $\begin{array}{l}\text { Positive Verknüpfung zu- } \\
\text { gunsten derjenigen, die den } \\
\text { Patienten den Blister anbieten }\end{array}$ & $\begin{array}{l}\text { Herausstellung der Anbieter als mo- } \\
\text { dern, sicherheitsinteressiert, patien- } \\
\text { tenfreundlich etc. }\end{array}$ \\
\hline Organisatorisch & $\begin{array}{l}\text { Orientierung an den Prä- } \\
\text { ferenzen der relevanten } \\
\text { Hauptakteure und den } \\
\text { Funktionsmechanismen } \\
\text { des Gesundheitssystems }\end{array}$ & Patienten, Ärzte & Krankenkassen \\
\hline
\end{tabular}

Quelle: Eigene Darstellung

\section{Kernelemente eines erfolgreichen Wochenblisterkonzeptes}

Vor dem Hintergrund der erst begonnen und dazu noch heterogenen wissenschaftlichen Beurteilung wurde die dargestellte Erhebung durchgeführt. Dafür wurde zur Beurteilung des Wochenblisterkonzeptes primär die Kosten-Nutzen-Perspektive der Kostenträger gewählt, da im Vollversorgungsansatz „Gesundes Kinzigtal“ partizipierende Krankenkasse und Managementgesellschaft gemeinsam durch eine einheitliche Einsparteilungslogik ${ }^{24}$ inzentiviert sind und in der traditionellen Arzneimittelversorgung einen Optimierungsbedarf vermuten. Nach Analyse der Erhebungsergebnisse, der Sichtung einschlägiger Literatur und der Befragung einzelner Ärzte und Patienten werden die folgenden Kriterien als erfolgsentscheidend für jedes industrielle Wochenblisterkonzept im deutschen Gesundheitssystem betrachtet:

\section{Inhaltlich/fachliche Ausprägung}

Inhaltlich sollte jedes Wochenblisterkonzept die festen und oral einzunehmenden Elemente der Gesamtmedikation älterer multimorbider Chroniker bedienen. Dabei sollte das Wochenblisterkonzept nicht an einzelnen Krankheiten, sondern am Krankheitsspektrum multimorbider Chroniker ausgerichtet werden. Notwendig ist es dafür, die für die Gesamtheit der Zielgruppe relevanten Wirkstoffe und Wirkstoffkombinationen zu identifizieren und im Sinne einer quantitativen und qualitativen Analyse zu gewichten. Diese Gewichtung und Koordination entspricht dann den Kernprinzipien optimierter Polipharmazie wie z.B. Prioritätensetzung bei der Behandlung von Krankheiten, beim Konsum von Arzneimitteln und bei der Festlegung von Einnahmezeitpunkten, wenn für den einzelnen Patienten gewährleistet bleibt, dass die für ihn notwendige Arzneimitteltherapie Anwendung fin- 


\section{THEMA}

den kann. Dafür ist es unter anderem erforderlich, keine notwendigen Wirkstoffe auszuschließen, unterschiedliche Wirkstoffstärken zuzulassen und auch die nicht festen bzw. oral einzunehmenden Arzneimittel im Medikationsplan zu berücksichtigen. Dies bedeutet auch, dass neben Generika auch Originalpräparate zu berücksichtigen sind, wo dies sinnvoll ist. Ein Wochenblister sollte nicht im Sinne einer limitierenden Positivliste, sondern im Sinne einer optimierten Gesamtmedikation konzipiert werden. Dies schließt nicht die Substitution einzelner Wirkstoffe der gleichen Wirkstoffgruppe/-klasse aus, um Wirkstoffe anderer Gruppen berücksichtigen zu können und dadurch das Spektrum behandelbarer Krankheiten und multimorbider Patienten insgesamt zu erweitern.

\section{Technische Ausprägung}

Um das mit dem Wochenblister verbundene inhaltliche Potenzial realisieren und diesen auf die individuellen Bedürfnisse der identifizierten Zielgruppe ausrichten zu können, ist es notwendig, die technische Realisierung des Wochenblisters an den folgenden Kernelementen auszurichten:

- Orientierung des Produktionsverfahrens an der Relevanz von Wirkstoffen und Wirkstoffstärken für die identifizierte Zielgruppe. Keine Limitierung in der Verwendung relevanter Arzneimittel als Folge des technischen Produktionsverfahrens.

- Eine für den Patienten übersichtliche Arzneimittelzusammenstellung in Form einer Matrixstruktur gegliedert nach Wochentagen und Einnahmezeitpunkten.

- Flexible Größe der Wochenblister in Abhängigkeit von der Anzahl der täglichen Tabletten - keine Wochenblistereinheitsgröße und keine unangemessene Beschränkung der Tablettenanzahl pro Tag.

- Flexible Anzahl und Terminierung der Einnahmezeitpunkte pro Tag (Keine durchgehende Begrenzung auf z.B. vier Einnahmezeitpunkte.)

- Möglichkeit, Hinweise zur Einnahme pro Tablette abzubilden wie z.B. vor, zu, nach dem Essen.

- Integration von Hinweisen auf die nicht feste bzw. oral einzunehmende Medikation.

- Transparente Wochenblisterstruktur, um den Patienten bzw. seinen Angehörigen oder prof. Pflegekräften eine „visuelle“ Möglichkeit der Selbstkontrolle und die vereinfachte Kommunikation mit dem Arzt zu ermöglichen.

- Altersgerechte Ausgestaltung des Wochenblisters mit ergonomischen Hilfen (Größe der Durchdrucklöcher, Schriftgröße ...).

- Perforation der Wochentage, um einzelne Abschnitte und nicht den ganzen Wochenblister „unterwegs“ mitnehmen zu können.
Darüber hinaus kommt es bei der technischen Ausprägung des Wochenblisters entscheidend auf den Aspekt der Arzneimittelsicherheit an. Der Wochenblister muss für jede Tablette denselben Sicherheitsstandard wie bei Fertigarzneimittel/Solida gewährleisten und damit die hohen im $\mathrm{AMG}^{25}$ definierten rechtlichen Anforderungen auch beim Produktions- und Verpackungsverfahren erfüllen. Für die Produktion und Herstellung industriell gefertigter Wochenblister bedeutet dies, dass sie unter Reinraumbedingungen erfolgen und dabei die GMP („Good Manufacturing Practices“) -Anforderungen der Pharmaherstellung vollständig eingehalten werden müssen. Für die Arzneimittel-Primärverpackung bedeutet dies z.B., dass sie die drei Grundfunktionen (umfassende und präzise Beschreibung des Inhaltes, Schutz vor Veränderungen durch Umwelteinflüsse während Lagerung, Transport und Handhabung sowie die schnelle, einfache und sichere Verwendung der Arzneimittel) gewährleisten muss. ${ }^{26} \mathrm{Zu}$ gewährleisten ist ihre Undurchlässigkeit für Luft, Licht (in Abhängigkeit vom einzelnen Produkt) und Feuchtigkeit sowie der Ausschluss von Abbau- und Wechselwirkungen der Arzneimittel untereinander und jeder Verwechslungsgefahr zwischen den Arzneimitteln. Zudem ist sicherzustellen, dass jede einzelne Tablette jederzeit identifizierbar und rückverfolgbar ist (Produkt, Hersteller, Wirkstoffstärke, verschreibender Arzt). Die Verpackung muss also zum einen die Vorzüge klassischer Arzneimittelverpackungen gewährleisten und zum anderen die Anforderungen an eine individuelle personenbezogene Arzneimittelzusammenstellung erfüllen.

Um diesen hohen Sicherheitsstandard erfüllen zu können, ist es im Wochenblisterkonzept notwendig, für jeden individuellen Patienten und jeden Applikationszeitpunkt die einzelnen Tabletten einzelverpackt, sortenrein und der ärztlichen Verordnung entsprechend zur Verfügung zu stellen und diese umfassend zu kennzeichnen (Wirkstoff, Hersteller...). Notwendig ist die Anwendung des UnitdoseSystems, um Arzneimittelsicherheit und Transparenz zu gewährleisten und damit im Unterschied zum MultidosePrinzip jede Form von Verwechslung, Untermischung und Querkontamination entnommener Tabletten auszuschließen. Zudem ist es notwendig, die für die Verwendung jedes Arzneimittels notwendigen Informationen (Beipackzettel) dem Patienten auf Grundlage der gesetzlichen Bestimmungen zusammen mit dem Wochenblister zur Verfügung zu stellen.

Neben der beschriebenen „technischen“ Arzneimittelsicherheit steht jedes Wochenblisterkonzept vor der Notwendigkeit, auch die durch Multimedikation entstehenden „Unsicherheiten“ z.B. in Bezug auf Interaktionen und Nebenwirkungen zu minimieren. Auch wenn die „technische" Arzneimittelsicherheit in Deutschland im internationalen Vergleich als hoch gilt, bestehen unter Experten Vorbehalte vor allem bezüglich der Interaktionssicherheit parallel verordneter Medikamente. So äußerte sich der Tagungsleiter Grandt zum Auftakt eines Fachkongresses für Patientensicherheit in der Arzneimitteltherapie am 30. November 2007 in dem Sinne, dass Arzneimitteltherapie vor allem bei älteren Menschen immer eine Hochrisikothe- 
rapie sei und diese überproportional von unerwünschten Wirkungen einer Arzneimitteltherapie betroffen sind. ${ }^{27}$ Der Staatssekretär im BMG, Schröder, äußerte sich beim selben Anlass in ähnlicher Weise und zog daraus den Schluss, dass das Thema Arzneimittelsicherheit in Zukunft noch aktiver angegangen werden müsse. ${ }^{28}$ Defizite in der Arzneimittelsicherheit werden in Deutschland also nicht im Bereich von Produktion, Herstellung oder Verpackung gesehen, sondern vor allem im Bereich der unkoordinierten Mehrfachmedikation oder durch Verwechslung beim Medikamentenstellen bzw. Ausgaben durch professionelles Pflegepersonal, auf Grund beispielsweise sehr ähnlichen Arzneimittelnamen bzw. -packungen ${ }^{29}$.

\section{Kaufmännische Ausprägung}

Letztlich sind die gesetzlichen Krankenkassen und soweit in der gleichen Anreizsituation auch IV-Systemgesellschaften die „Abnehmer“ des Produkts Wochenblister. Die kaufmännische Ausprägung jedes Geschäftsmodells hat sich demnach besonders mit deren Situation zu beschäftigen, darf dabei aber die Perspektive von Patienten, Ärzten und Apotheken nicht aus dem Blick lassen. Auf die spezifischen ökonomischen Anforderungen an Wochenblister wird im weiteren Verlauf unter dem Gliederungspunkt „Perspektive der Kostenträger“ detailliert eingegangen. Relevant ist an dieser Stelle vor allem, dass jedes Wochenblisterkonzept von den Kostenträgern nur dann nachgefragt werden dürfte, wenn es im Vergleich zur traditionellen Arzneimittelversorgung kosteneffektiv ist bzw. andere Vorteile im Wettbewerb bringt. Zu berücksichtigen ist dabei vor allem der spezifische Rahmen der Möglichkeiten der Rabattverträge nach $\S 130$ a Abs. 8. Der Anbieter eines Wochenblisters muss sich nicht nur an den üblichen N3-Preisen der chronischen Medikation, sondern auch an den durch die Rabatte der Generikahersteller noch einmal abgesenkten Preise messen lassen. Jeder Anbieter steht damit vor der Notwendigkeit, den Nutzen seines Produktes zu spezifizieren, monetär zu bewerten und ggf. Garantien für das Eintreten prognostizierter Effekte zu geben. Aus der Perspektive der Kostenträger kommt es dann entscheidend darauf an, dass der in Aussicht gestellte Nutzen die für den Kostenträger anfallenden Kosten mindestens kompensiert.

\section{Organisatorische Ausprägung}

Um das mit dem Wochenblister verbundene Potenzial zu realisieren, kommt es neben den beschriebenen inhaltlichen, technischen und kaufmännischen Ausprägungen entscheidend darauf an, den Wochenblister so in die Funktionsmechanismen des Gesundheitssystems einzubauen, dass er von den Akteuren auch angewendet wird. Dabei ist vor allem die Perspektive der Patienten, Ärzte, Kostenträger und Apotheker von Bedeutung. Jede Wochenblisterkonzeption, die sich auf inhaltliche und technische Aspekte beschränkt und die Präferenzen der relevanten Akteure nicht ausreichend berücksichtigt, wird in der Umsetzung scheitern. Wie sieht nun die Perspektive der einzelnen Akteure aus?

\section{Die Perspektive der Patienten}

Den Patienten fällt als Endverbraucher insbesondere im häuslichen Umfeld eine zentrale Rolle bei der Nachfrage nach Wochenblistern zu. In einer Zeit, in der der Gesetzgeber die Entscheidungsmacht der Patienten durch verschiedene Wahloptionen (siehe im SGB V die $\S \S 53,73 \mathrm{~b}, \mathrm{c}$ und 140ff.) zunehmend aufwertet, kann den Patienten mit dem Wochenblisterkonzept eine echte Alternative im Bereich der Arzneimittelversorgung angeboten werden. Dabei kommt es vor allem unter dem Aspekt der Therapietreue von Chronikern entscheidend darauf an, dass sich Patienten freiwillig und ggf. gemeinsam mit dem Hausarzt für oder gegen das Konzept entscheiden.

Die Analyse der eigenen Erhebungen sowie erste Befragungen von den im IV - Projekt eingeschriebenen Patienten haben gezeigt, dass es den Patienten der spezifischen Zielgruppe bei ihrer Entscheidung für oder gegen ein Wochenblisterkonzept vor allem auf die folgenden Aspekte ankommt:

- Orientierung in der Arzneimitteltherapie mit ärztlicher und pharmazeutischer Unterstützung ohne unangemessene Einschränkung der Therapiefreiheit.

- Möglichkeit, das Wochenblisterkonzept auch wieder verlassen zu können..

- Freiheit bei der Wahl des Bezugs.

- Convenience-Gewinn durch Reduzierung des Aufwandes beim eigenen „Stellen“ und „Einnehmen“ der Medikation, „einfache“ Handhabung (große Schriftgröße, bequemer Ausdruck...).

- Sicherheit, Transparenz und Dokumentation der verwendeten Arzneimittel.

\section{- Abstimmung mit dem behandelnden Arzt.}

Wichtig ist den meisten Patienten der Zielgruppe neben einer freiwilligen Entscheidung für oder gegen das Konzept somit vor allem eine verbesserte Abstimmung einzelner Therapieelemente und ein eigener „Convenience-Gewinn“. Die Vermeidung einer erhöhten Eigenbeteiligung wurde von einzelnen der befragten Patienten ebenfalls benannt. Inwieweit dieser Wunsch ein hartes Nachfragekriterium darstellt, konnte nicht festgestellt werden, zumal belastbare Studien über die Zahlungsbereitschaft der Zielgruppe genauso fehlen wie Aussagen über eine mögliche Preiselastizität der Nachfrage. Vermutet wird, dass die Zahlungsbereitschaft der Patienten primär von dem durch den Wochenblister erzielbaren Convenience-Gewinn abhängt.

\section{Die Perspektive der Ärzte}

Ärzte nehmen beim Arzneimittelkonsum generell eine Schlüsselrolle ein. Sie entscheiden über Diagnose und Therapie und wählen die zu verordnenden Arzneimittel bzw. Wirkstoffe aus. Durch ein vertrauensvolles Arzt - Patienten - Verhältnis verfügen sie zudem über einen zentralen Einfluss auf das Patientenverhalten. Im Bereich der PAV kommt den Ärzten zudem eine zentrale Rolle zu, weil es ihnen obliegt, PAV bzw. Wochenblister zu verordnen und 


\section{THEMA}

gemeinsam mit dem Patienten die freiwillige Teilnahme zu vereinbaren.

In der eigenen Erhebung wurden Allgemeinärzte und Internisten als die für die Zielgruppe mit Abstand relevantesten Arztgruppen identifiziert. Für beide Arztgruppen stellen ältere multimorbide Chroniker einen Großteil der eigenen Patienten dar. Für beide Arztgruppen ist die Versorgung multimorbider Chroniker aber traditionell mit viel Aufwand verbunden. Analysen des Verordnungsverhaltens einzelner Ärzte lassen zudem eine nicht zu vernachlässigende Verordnungsheterogenität bei der Therapie multimorbider Chroniker vermuten.

Die Analyse der eigenen Erhebungen sowie erste Befragungen von Netzärzten haben gezeigt, dass es den Allgemeinärzten und Internisten bei ihrer Entscheidung für oder gegen ein Wochenblisterkonzept vor allem auf die folgenden Aspekte ankommt:

- Aufwertung der Steuerungsfunktion hausärztlicher Tätigkeit

- Patienten- statt Krankheitsbezug in der Arzneimittelversorgung

- Freiwillige Teilnahme des Patienten; kein Patientenverlust

- Keine zusätzliche Belastung des Arzneimittelbudgets

- Kein zusätzlicher organisatorischer Aufwand

Bei der konkreten Ausgestaltung der Wochenblisterkonzeption kommt es beiden Arztgruppen besonders auf die folgenden Aspekte an:

- Verbesserte Strukturierung und Koordination der Arzneimitteltherapie multimorbider Chroniker.

- Wochenblister als Orientierungs- und Optimierungshilfe bei der Arzneimitteltherapie multimorbider Chroniker und nicht als Einschränkung. Gewährleistung hoher Qualitätsstandards.

- Hohe Übersichtlichkeit des Wochenblisters für den Patienten, um die Kommunikation zwischen Arzt und Patienten zu erleichtern.

- Beitrag zur Erhöhung der Verordnungssicherheit z.B. durch EDV - gestützte Interaktionschecks.

- Einfachheit der Lösung z.B. durch eine elektronische Anbindung an die bestehende Praxis-EDV, Verzicht auf ein eigenständiges System, Vermeidung von Zusatzaufwand im Praxisablauf (oder ggf. alternativ eine angemessene Vergütung dieses Zusatzaufwands).

- Möglichkeit zur Entlastung des eigenen Arzneimittelbudgets z.B. durch die Vermeidung unabgestimmter Verordnungen.

Es kommt aus der Arztperspektive also vor allem darauf an, den Wochenblister als organisatorische und inhaltliche Unterstützung im Praxisalltag zu konzipieren und dabei die ärztliche Therapiefreiheit nicht unangemessen einzuschränken sowie das arztspezifische Arzneimittelbudget nicht zusätzlich zu belasten. Die mit der Vergütungsreform 2009 geplante stärkere Orientierung der ärztlichen Vergütung an der real vorhandenen Morbidität lässt zudem ein stärkeres monetäres Interesse der Ärzte an der Behandlung multimorbider Chroniker und damit an der Zielgruppe der Wochenblister erwarten.

\section{Die Perspektive der Kostenträger}

Die gesetzlichen Krankenkassen stehen untereinander in einem zunehmenden Wettbewerb. Mit der für 2009 geplanten Einführung des Gesundheitsfonds stehen sie zudem vor der Notwendigkeit eines umfassenden Kostenmanagements, um keine Zusatzprämie erheben zu müssen. In dieser Situation werden alle Ausgaben auf ihre Notwendigkeit überprüft, Investitionen mit unklarem bzw. erst langfristig erwartetem Nutzen müssen durch exakte Kosten-Nutzen-Rechnungen hinterlegt werden. Durch die Morbiditätsorientierung des Gesundheitsfonds erhalten die gesetzlichen Krankenkassen ab dem Jahr 2009 zudem einen starken Anreiz, die Gesamtversorgung mit fokussiertem Blick auf die relevanten chronischen Krankheiten zu optimieren. Im Kassenwettbewerb werden in der unmittelbaren Zukunft somit relative Kostenvorteile in der Chronikerversorgung erfolgsentscheidend. Entsprechend werden die Kostenträger auch von den Leistungserbringern zunehmend eine kosteneffektive Versorgung (multimorbider) Chroniker einfordern.

Vor diesem Hintergrund stellen die Kostenträger an alle neuen Produkte und Dienstleistungen drei zentrale Anforderungen:

- (Kurzfristiges) Preisbewußtsein und Vermeidung von Kostensteigerungen. Konkret darstellbarer, monetär bewertbarer und möglichst bereits kurzfristig eintretender Nutzen.

- Beitrag zur kosteneffektiven Versorgungsoptimierung insbesondere auch bei den ausgleichsrelevanten chronischen Krankheiten.

- Unterscheidungsmöglichkeit im Wettbewerb, Instrument der Kundenbindung und -gewinnung.

Die Kostenträger werden auch den Wochenblister vor allem danach beurteilen, ob und in welchem Maße er einen Beitrag zur Erfüllung dieser drei zentralen Anforderungen leisten kann. Aus der Perspektive der Kostenträger kann das von Lauterbach et al. ${ }^{30}$ bzw. Wille \& Wolff ${ }^{31}$ diskutierte „Geschäftsmodell Wochenblister“ deshalb noch nicht umfänglich überzeugen, weil sicher anfallenden $\mathrm{Zu}$ satzkosten in Höhe von genannten ca. $3 €$ pro Wochenblister kein entsprechender, kurzfristig eintretender und konkret monetär bewertbarer Nutzen gegenüber steht. Nach eigener Einschätzung entspricht der von Lauterbach et al. ${ }^{32}$ diskutierte Ansatz den Kostenträgerpräferenzen konkret in den folgenden Aspekten noch nicht ausreichend:

- Konkreten Zusatzkosten steht ein unkonkreter Nutzen gegenüber. 
- Eine durch den Wochenblister steigende Compliance kann ebenso wie evtl. daraus resultierende monetäre Effekte nicht ausreichend spezifiziert werden.

- Die erwartete Kosteneinsparung durch die bevorzugte Verwendung von Generika dürfte geringer ausfallen als erwartet, da die definierte Zielgruppe ohnehin stark auf Generika eingestellt ist und deren Preise von den Krankenkassen zunehmend über Rabattverträge verhandelt werden.

- Ein tablettenspezifischer Referenzwert auf Basis der günstigsten verfügbaren Packungsgröße (primär N3) führt nur eingeschränkt zu einer Kostenersparnis, da viele Patienten der Zielgruppe ohnehin auf N3 - Packungen eingestellt sind.

- Ein reduzierter Arzneimittelverwurf wird bei der Zielgruppe der stabil eingestellten Chroniker gering ausfallen.

- Nachgelagerte Kosteneinsparungen können für das deutsche Gesundheitssystem nicht belegt werden. Die aus den USA beispielhaft dargestellte Verweildauerreduzierung im Krankenhaus ist unter DRG - Bedingungen in Deutschland ohne Relevanz für die Kostenträger.

Um das Interesse der Kostenträger in Deutschland zu wecken, kommt es nach eigener Einschätzung vielmehr darauf an, mit dem Wochenblisterkonzept einen konkreten Mehrnutzen für die Kostenträger zu generieren. Aus diesem Grund sollte jede Konzeption vor allem an den folgenden Elementen ausgerichtet sein:

- Kostenneutralität für die Kostenträger in der Arzneimittelversorgung als oberste Zielvorstellung - wie im Abschnitt Kaufmännische Ausprägung bereits erwähnt unter Einbeziehung der Rabattvorteile, die Kostenträger mit Arzneimittelherstellern abgeschlossen haben.

- Berücksichtigung der im Gesundheitsfonds ausgleichsrelevanten Krankheiten und der Hauptzielgruppe der multimorbiden Chroniker.

- Abbau von Fehl- und Überversorgung in Bezug auf Arzneimittel durch eine mit den einzelnen Kostenträgern abgestimmte und an den Kernprinzipien effektiver Polipharmazie orientierte „Bestückung“ der Wochenblister.

- Berücksichtigung der bevorzugten Steuerungsinstrumente im Arzneimittelmarkt wie Orientierung an bestehenden Leitlinien, Rabattverträge und Zuzahlungsbefreiungen. Einbindung in Neue Versorgungsformen wie Hausarztverträge, DMP und Integrierte Versorgung.

- Kostenträger- oder kassenartenspezifische Einzelverträge mit Berücksichtigung spezifischer Präferenzen und eigener Gestaltungsoptionen der Kostenträger.

- Bereitschaft zu einer am Erfolg orientierten Vergütungsform („value“- und „shared risk“ - Orientierung der Verträge).

Neben dem ökonomischen Aspekt soll aber auch auf organisatorische Aspekte hingewiesen werden. So haben
Kostenträger ein direktes Interesse an der Minimierung von Transaktionskosten und Verfahrenskomplikationen. Preisvereinbarungen und Abrechnungsprozeduren sollten so möglichst den bisher bekannten Formen folgen bzw. besonders unaufwändig und mit möglichst wenigen ITÄnderungen controllingfähig sein.

\section{Die Perspektive der Hersteller/ Apotheken}

Die meisten Arzneimittelhersteller und Apotheken betrachten multimorbide Chroniker als ihre Hauptkundengruppe. Der auf diese Zielgruppe zugeschnittene Wochenblister bietet ihnen einen neuen Absatzkanal von zentraler Bedeutung und stellt gleichzeitig vor allem für die Apotheken ein wichtiges Instrument zur Kundenbindung dar. Beide Gruppen werden in jedem Wochenblisterkonzept zwingend benötigt. Die pharmazeutische Kompetenz der Apotheken wird vor allem bei der abgestimmten Zusammenführung der Verordnungen und in Kooperation mit den relevanten Ärzten in der Überprüfung der Medikation auf Doppelmedikationen und Wechselwirkungen benötigt. Zudem spielt die Apotheke bei der Ausgabe der Wochenblister eine zentrale Rolle. Ebenso wie in der Arztpraxis kommt es auch in der Apotheke auf die Vermeidung eines organisatorischen Mehraufwandes und die Einfachheit der Lösung z.B. durch eine elektronische Anbindung an die bestehende Apotheken-EDV und den Verzicht auf ein eigenständiges System an. Welche Rolle Apotheken und Hersteller bei der Herstellung des Wochenblisters spielen, hängt vom spezifischen Wochenblisterkonzept ab. Für die Hersteller stellt jedes Wochenblisterkonzept einen zukunftsträchtigen Absatzkanal dar. Da im Sinne einer optimierten Polipharmazie aber nicht alle denkbaren Wirkstoffe für ein Wochenblisterkonzept benötigt werden, stehen die Hersteller vor der Herausforderung, ihre Arzneimittel in den einzelnen Wochenblistern zu platzieren.

Es kommt zusammenfassend darauf an, bei jedem Wochenblisterkonzept die Präferenzen der relevanten Akteure zu berücksichtigen und einen konkreten Nutzen für jede einzelne Zielgruppe zu generieren. Dieser besteht bei entsprechender Gestaltung für die einzelnen sich im Wettbewerb befindlichen Kostenträger vor allem in einem ökonomischen Vorteil und einer langfristigen Bindung vor allem der Versicherten mit ausgleichsrelevanter chronischer Morbdität. Gerade für diese Zielgruppe bietet ein entsprechend konzipierter Wochenblister den Kostenträgern ein ideales Steuerungsinstrument im Bereich der Arzneimitteltherapie. Patienten bzw. ihre Angehörigen werden sich dann für ein Wochenblisterkonzept entscheiden, wenn es die Einnahme der regelmäßig zu konsumierenden Arzneimittel unterstützt, die Gesamtmedikation optimal abgestimmt wird und der behandelnde Arzt das Wochenblisterkonzept mit trägt. Die Ärzte wiederum werden vor allem Wert darauf legen, dass sie weiterhin die Arzneimitteltherapie bestimmen und durch einen Wochenblister nicht entmündigt, sondern inhaltlich und organisatorisch unterstützt werden. Für die Apotheken muss der Hauptnutzen darin bestehen, die Hauptkundengruppe der Arzneimittelchroniker an sich zu binden. 


\section{Wie sind vor diesem Hintergrund die gegenwärti- gen Entwicklungen in Deutschland zu beurteilen?}

In Deutschland werden in der gegenwärtigen Diskussion die manuelle und die industrielle Herstellung von Wochenblistern diskutiert. Während die manuelle Verblisterung vorwiegend in Apotheken stattfindet, ist eine industrielle Verblisterung nur unter Reinraumbedingungen und unter vollständiger Einhaltung der GMP („Good Manufacturing Practices“) - Anforderungen der Pharmaherstellung denkbar.

Variante 1: Manuelle bzw. automatisierte Verblisterung in Apotheken

Die Verblisterung in der niedergelassenen Apotheke erfolgt in aller Regel manuell, manchmal mit Hilfe von Apparaturen durch „Auseinzeln“ der benötigten Arzneimittel aus konventionellen Verpackungen (N1 - N3). Damit stellt diese Variante der Verblisterung immer einen zusätzlichen Prozess bei Beibehaltung der traditionellen Arzneimitteldistribution dar. Die Neu-Verblisterung erfolgt anschließend von Hand oder z.B. mit Hilfe von Schlauchbeutelautomaten. Verblistert wird heute vor allem in städtischen Regionen mit regionalem Apothekenwettbewerb mit dem Ziel, die Zielgruppe der Chroniker an die eigene Apotheke zu binden. Zu diesem Zweck wird den Patienten (oft in Alten- und Pflegeheimen) der Wochenblister in aller Regel in Form von Multidose-Verpackungen von den betreffenden Apotheken kostenlos als zusätzliches „Convenience - Produkt“ zur Verfügung gestellt. Für die Kostenträger entstehen bei dieser Art der Verblisterung keine zusätzliche Kosten, aber auch keine zusätzlichen Effekte z.B. in Form niedrigerer Arzneimitteleinkaufspreise, gesteigerter Effizienz in der Arzneimitteldistribution oder einem größeren Einfluss auf die Arzneimitteltherapie. Ärzte sind an dieser Form der Verblisterung in aller Regel nicht beteiligt. Alle bisher bekannten Verblisterungsarten in Apotheken stellen vornehmlich Insellösungen für die eigene Apothekenkundschaft ohne einen flächendeckenden Anspruch dar. Sie haben nur unter der Voraussetzung einer auch wirklich abgestimmten Medikation das Potenzial, lokal die Arzneimittelversorgung zu verbessern. Ihnen sind aber vor allem in Bezug auf Flächendeckung, Kosteneinsparungen für die Kostenträger und Aspekte der Arzneimittelsicherheit klare Grenzen gesetzt. So darf eine Apotheke nur die eigenen Patienten bzw. Pflegeheime mit einem Versorgungsvertrag bedienen. Die Versorgung der Patienten einer anderen Apotheke ist nicht zulässig. Hierfür ist eine Herstellungserlaubnis nach dem AMG notwendig. Die Verblisterung wird in der Regel aus der Marge der in der Arzneimittelpreisverordnung festgelegten Aufschläge auf rezeptpflichtige Fertigarzneimittel finanziert.

\section{Variante 2: Industrielle Verblisterung}

Zum gegenwärtigen Zeitpunkt gibt es in Deutschland vier Unternehmen, die mit einer Herstellererlaubnis ausgestattet den Apotheken bundesweit eine industrielle patientenindividuelle Verblisterung als Dienstleistung anbieten. Drei der Anbieter nutzen zum Verblistern eine große Anzahl an Schlauchbeutelautomaten. Ein Unternehmen hingegen verpackt die Tabletten in einer standardisierten Blisterkarte mit 7x4 Multidose-Blisterhöfen.

Auch wenn die Vorteile einer industriellen Verblisterung gegenüber der manuellen Variante evident sind (kein zwingend redundanter Verpackungsprozess, Arzneimittelsicherheit, Möglichkeit flächendeckender Versorgung, Verhandlungspotenzial beim Tabletteneinkaufspreis, Potenzial für Evaluationen...), besteht die Notwendigkeit für jeden Anbieter darin, die industrielle und wochenspezifische Verblisterung vertragsrechtlich so zu verankern, dass sie den Kostenträgern einen konkreten Mehrnutzen bietet und so in die Funktionsmechanismen des Gesundheitssystems eingebunden wird, dass es den Präferenzen der relevanten Akteure entspricht. Notwendig dafür sind konkret:

- Individueller $\S 130$ a Vertrag - evtl. mit „value - Bezug“ - zwischen Anbieter und Krankenkasse bzw. Anbieter und von Krankenkassen beauftragter Managementgesellschaft orientiert an dem Kernelement einer Kostenneutralität für die Kostenträger. Da der erwirkte Gesamtnutzen eines Wochenblistereinsatzes ex ante weder aus der Literatur noch aus anderen Rechnungen valide vorhersagbar ist, sollten Kostenträger und Anbieter sinnvollerweise den möglichen Nutzen und das mögliche Risiko teilen. Hierfür könnte man sich der in den letzen Jahren entwickelten „Shared Value - Shared Risk“ Vertragsmodelle ${ }^{33}$ bedienen.

- Die bei einem fehlenden konkreten Zusatznutzen vermutete fehlende Zahlungsbereitschaft von Kostenträgern und Patienten führt für den Anbieter automatisch zu der Notwendigkeit, andere Finanzierungsquellen zur Kostendeckung zu erschließen.

- Um das mit dem Wochenblister verbundene Potenzial realisieren und den Aufwand für die beteiligten Ärzte und Apotheken je Einheit minimieren zu können, sollte langfristig eine Flächendeckung vereinbart werden.

- Einschreibemodell für alle interessierten Patienten. Patienten sollten weder vom Gesetzgeber, noch vom Kostenträger oder dem behandelnden Arzt in ein Wochenblisterkonzept gezwungen werden.

- Ärzte und Apotheker müssen die Möglichkeit erhalten, an dem Modell zu partizipieren. Jedes Wochenblisterkonzept sollte allen interessierten Ärzten und Apothekern offen stehen. Dies kann insbesondere in Form der Neuen Versorgungsformen geschehen. Um beide Berufsgruppen für das Konzept zu gewinnen, sind ihre oben dargestellten Präferenzen bei der konkreten Konzeption und Vertragsgestaltung zu berücksichtigen. Eine darüber hinaus gehende Vergütung für ihre Teil- 
nahme würde diese Bereitschaft sicherlich noch weiter fördern, erscheint bei den budgetierten Finanzmitteln der Kostenträger und der eingeschränkten individuellen Zahlungsbereitschaft der Patienten aber kaum möglich.

\section{Fazit und Ausblick}

Der Wochenblister birgt für die Kostenträger und das Gesundheitssystem ein deutlich größeres Nutzenpotenzial als bisher in der öffentlichen Diskussion artikuliert wird. Die optimal dafür geeignete Zielgruppe besteht deutschlandweit aus mindestens 6,4 Millionen zum großen Teil multimorbiden und älteren „Arzneimittelchronikern“ mit sogar langfristig steigender Tendenz.

Das ökonomische Potenzial eines Wochenblisters für die Kostenträger und das Gesundheitssystem besteht nur partiell aus einzelnen und noch nicht abschließend erforschten Komponenten wie einer Compliancesteigerung, einem reduzierten Verwurf etc., zumal jede Wochenblisterkonzeption diese Aspekte nur partiell beeinflussen kann. Die beiden wichtigsten ökonomischen Potenziale industriell gefertigter Wochenblister bestehen konkret in den verhandelbaren Arzneimitteleinkaufspreisen und der Anwendung der Kernprinzipien von Polipharmazie. Durch die Unabhängigkeit der PAV von der Arzneimittelpreisverordnung und die Option einer Anbindung an Rabattverträge nach $\$ 130$ a entsteht unter der Voraussetzung, dass relevante Mengen gebündelt und verbindlich nachgefragt werden, ein relevantes Verhandlungspotenzial bei den Arzneimitteleinkaufspreisen. Die Anwendung der Kernprinzipien optimierter Polipharmazie bei der abgestimmten Medikationszusammenführung lässt einen Abbau vor allem von Über- und Fehlversorgung in der Arzneimitteltherapie multimorbider Chroniker erwarten. Zudem gehen viele Experten davon aus, dass gerade in der optimierten Gesamtmedikation multimorbider Chroniker ein erhebliches Potenzial auch zur Reduzierung nachgelagerter Kosten besteht.

Im Unterschied zu anderen formulierten ökonomischen Zielen können beide Ziele durch ein entsprechend abgestimmtes Wochenblisterkonzept allein und damit weitgehend unabhängig von anderen Faktoren erreicht werden. Auch das Ziel einer Compliancesteigerung kann im Wochenblisterkonzept offensichtlich am ehesten dadurch erzielt werden, dass dem Patienten durch die intendierte Medikamentenkoordination die Angst vor Interaktionen und Nebenwirkungen genommen wird. ${ }^{34}$ Zudem wird von einer abgestimmten Gesamtmedikation auch ein nicht unerheblicher Effekt auf die Arzneimittelsicherheit erwartet. ${ }^{35}$

Wegen der gegenwärtig und absehbar begrenzten Finanzmittel der Kostenträger springt jedes Konzept zu kurz, das zur Gegenfinanzierung auf zusätzliche Einnahmen der Kostenträger angewiesen ist, wenn keine im Vergleich zu den Rabattverträgen der Krankenkassen günstigeren Einkaufsoptionen erzielt werden können bzw. keine ökonomische Ergebnisverantwortung übernommen wird.
Die industrielle Verblisterung bietet im Vergleich zur manuellen Verblisterung das Potenzial, flächendeckende Lösungen zu konzipieren, niedrigere Arzneimitteleinkaufspreise zu erzielen, die Arzneimittelsicherheit zu erhöhen (nur bei Anwendung des Unitdose-Prinzips) und den Arzneimittelverbrauch (vor allem mit der Perspektive der Nutzung der elektronischen Patientenakte) transparenter zu gestalten. Diese technischen Vorteile einer industriellen Verblisterung kommen aber nur dann zum Tragen, wenn der Wochenblister inhaltlich, kaufmännisch, organisatorisch und vertraglich angemessen implementiert wird. Dabei kommt es auf der Seite der Anbieter entscheidend auf die Produktion eines konkreten „Mehrnutzens“ an. Ein Wochenblisterkonzept ohne konkreten Zusatznutzen und value-Bezug sowie ohne die ausreichende Berücksichtigung der Präferenzen von Patienten, Ärzten, Herstellern und Apothekern wird an den limitierten Finanzmitteln der Kostenträger und der eingeschränkten Zahlungsbereitschaft der Zielgruppe scheitern.

Aus Sicht der Kostenträger ist strategisch vor allem relevant, dass der Wochenblister ihnen bei einer entsprechenden Vertragsgestaltung im Unterschied zu der traditionell ausgeübten Praxis der nachgelagerten Kostenprüfung die Möglichkeit bietet, durch die Auswahl und Bündelung relevanter Wirkstoffe aktiven Einfluss auf die Gesamtmedikation multimorbider Chroniker zu nehmen. Hinzu kommt, dass sich ein industriell gefertigter Wochenblister für jeden Kostenträger strategisch auch als Marketingplattform eignet.

Die Gesundes Kinzigtal GmbH verspricht sich von einem entsprechenden Wochenblisterkonzept einen relevanten ökonomischen Effekt. Wir konnten feststellen, dass der Wochenblister für ca. 8\% der Versicherten eine Lösungsoption darstellt. Damit wären im Modellprojekt bei durchschnittlichen Arzneimitteltherapiekosten der relevanten Zielgruppe von ca. $1.100 €$ p.a. und Patient mehr als 2,5 Mio. $€$ der Arzneimitteltherapiekosten im unmittelbaren Fokus des Wochenblisterkonzeptes. Selbst ein aufgrund des oben beschriebenen wirtschaftlichen und medizinischen Nutzenpotenzials vorsichtig geschätztes Einsparvolumen von $10 \%$ würde bei einem kostenneutralen Wochenblistereinsatz zu einem jährlichen Einsparbetrag von ca. 250.000 $€$ führen. Um dieses Einsparvolumen auch realisieren zu können, kommt es im nächsten Schritt darauf an, das Konzept detailliert weiter zu entwickeln und konkrete Modellvorhaben zu implementieren.

\section{Literatur}

- $\quad$ AOK Bundesverband (2007): Risiken erkennen - Fehler vermeiden: www.aok-bv.de/gesundheit/themen/index_13031.html

- Arzneimittelpreisverordnung (APV) (November 2007): www.gesetzeim-internet.de/ampreisv/BJNR021470980.html

- Cohen (2006): New Breed of PBMs are poised to address transparency in healthcare: Managed Healthcare Executive, February 1, 2006: www. partnersrx.com/_downloads/Pharmacy-Best-PBMs.pdf

- Düsing (2006): Medikamentöse Therapie mit verblisterten Arzneimitteln: Möglichkeiten und Chancen, Bonn

- Edlavitch/Salmon (2006): Point of View - Drug Safety within Drug Use: Disease Management, Volume 9, Number 5, 2006, 259-265

Foxbusiness (2008): Pharmacists Customize Prescriptions by Patient, Day and Dose with onePAC ${ }^{\text {m }}$ Packaging: www.foxbusiness.com/arti- 
cle/pharmacists-customize-prescriptions-patient-day-dose-onepactmpackaging_493081_1.html

- Gerste/Niemeyer/Lauterberg (1999): Wieviele chronisch Kranke gibt es? - Annäherung mit Hilfe einer Analyse von Routinedaten, in: Arnold/ Litsch/Schwartz (Hrsg.): Krankenhausreport 1999; Schwerpunkt: Versorgung chronisch Kranker, Stuttgart

- Gesetz über den Verkehr mit Arzneimitteln AMG (letzte Änderung 01.08.2007): www.gesetze-im-internet.de/amg_1976/index.html

- Gesetz zur Stärkung des Wettbewerbs in der gesetzlichen Krankenversicherung (GKV- Wettbewerbsstärkungsgesetz - GKV-WSG (2007): www. die-gesundheitsreform.de/gesetze_meilensteine/gesetze/gesund-heitsreform_2007/index.html

- Glaeske in Kooperation mit Pharmafacts (2007): Analyse der Verordnungen in einer GKV-Population im Hinblick auf die Eignung für eine individuelle Verblisterung, Berlin

- Hermann/Hildebrandt/Richter-Reichhelm/Schwartz/Witzenrath (2006): Das Modell „Gesundes Kinzigtal“- Managementgesellschaft organisiert Integrierte Versorgung einer definierten Population auf Basis eines Einsparcontractings, erschienen in: Gesundheits- und Sozialpolitik, Heft 5/6, Mai/Juni 2006, 60. Jahrgang, Baden-Baden

- Hildebrandt/Brunn/Niedermaier/Rothe et.al. (2007): Neue Ideen für Neue Player - Rabattverträge \& Co. verändern die Pharma-Industrie nachhaltig, erschienen in: pharma relations, 08/07, eRelation AG, Bonn

- Kane/Brixner/Rubin/Sewitch (2008): The Challenge of Compliance and Persistence: Focus on Ulcerative Colitis, erschienen in: JMCP Journal of Managed Care Pharamcy, Supplement, Volume 14, Nr. 1, 2008

- Krämer (2005): ADKA-Statement: Einzeldosis-Blisterverpackung je abgeteilte Arzneiform, in: Krankenhauspharmazie 26. Jahrgang, Nr.3

- Kuhlmey et al. (2005): Versorgungsverläufe bei chronisch kranken älteren Menschen: Eine patientenorientierte Analyse zur bedarfsgerechten und wirtschaftlichen Steuerung des Versorgungsgeschehen - Forschungsbericht, Berlin

- Lauterbach/Gerber/Lüngen (2004): Internationale Erfahrungen mit der Verblisterung von Arzneimitteln, Endbericht, Köln

- Lauterbach/Lüngen/Gerber (2004): Nutzung der Verblisterung von Arzneimitteln im Rahmen von Disease-Management-Programmen, Endbericht, Köln

- Lauterbach/Gerber/Stollenwerk/Lüngen (2006): Verblisterung von Arzneimitteln für Bewohner von Alten- und Pflegeheimen und in der häuslichen Pflege: Beschreibung und Bewertung eines Pilotprojekts (September 2004 bis Dezember 2005), Endfassung, Köln

- Lauterbach/Lüngen/Gerber (2006): Auswirkungen des Einsatzes von individualisierten Blistern auf Kosten und Qualität der Arzneimitteltherapie, Köln

- Lauterbach/Lüngen/Gerber/Kohaupt/Büscher (2007): Quantifizierung der Fehlwurfrate beim Stellen fester, oraler Darreichungsformen in drei Pflegeheimen, Endfassung, Köln

- Lee/Grace/Taylor (2006): Effect of a Pharmacy Care Program on Medication Adherence and Persistence, Blood Pressure, and Low Densitiy Lipoprotein Cholesterol - A Randomized Controlled Trial, JAMA Volume 296, Nr. 21, 2563-2571

- Nair/Salmon/Kaul (2007): Iatrogenic Disease Mangement: Moderating Medication Errors and Risks in a Pharmacy Benefit Management Environment, in: Disease Management, Volume 10, Nr. 6, 2007, 337-346

- Schaeffer/Müller-Mundt/Haslbeck (2007): Bewältigung komplexer Medikamentenregime bei chronischen Erkrankungen - Herausforderungen aus der Sicht der Gesundheitsprofessionen, Bielfeld
- Søndergaard/Gundgaard/Sørensen/Hansen (2006): Dose-dispensed medicine and associated costs of medicine and health care, Register-based-analysis, Kopenhagen

- $\quad$ SVRKAG (Hrsg.) (2000/2001): Bedarfsgerechtigkeit und Wirtschaftlichkeit - Zur Steigerung von Effizienz und Effektivität der Arzneimittelversorgung in der gesetzlichen Krankenversicherung (GKV), Addendum zum Gutachten 2000/2001,

- Statistisches Bundesamt (2007), www.destatis.de

- White (2008): More prescriptions, greater confusion: www.baltimoresun.com/news/health/bal-te.md.meds09mar09,0,85144. story?page $=1$

- Wille/Wolff (2006): Verblisterung von Arzneimitteln, Gutachten im Auftrag des Verbandes Forschender Arzneimittelhersteller e.V. (VFA), Endbericht

\section{Fußnoten}

1 Das Gutachten des wissenschaftlichen Beirats des Bundesversicherungsamtes wurde im Januar 2008 den Spitzenverbänden der Krankenkassen vorgelegt und die Anhörungen dazu eingeleitet.

2 Vgl. Lauterbach et al. (2004); (2006); (2007)

3 Vgl. Lee/Grace/Taylor (2006)

4 Vgl.Kuhlmey et al. (2005)

5 Vgl. Statistisches Bundesamt (2007)

6 Vgl. Wille/Wolff (2006)

7 Vgl. Gerste/Niemeyer/Lauterberg (1999)

8 Vgl. Glaeske (2007)

9 SVRKAG $(2000 / 2001)$

10 Vgl. Schaeffer et al. (2007)

11 Vgl. Lauterbach/Gerber/Lüngen (2006)

12 ebd.

13 Vgl. Lee/Grace/Taylor (2006)

14 Vgl. Lauterbach et al. (2004); (2006); (2007)

15 Vgl. Düsing (2006)

16 Vgl. Wille/Wolff (2006)

17 Vgl. Glaeske (2007)

18 Heute: $7 x 4$ Pharma GmbH

19 Vgl. Lauterbach/Gerber/Stollenwerk/Lüngen (2006)

$20 \mathrm{Vgl}$. Wille/Wolff (2006)

21 ebd.

22 Vgl. Düsing (2006)

23 Vgl. Glaeske (2007)

24 Vgl. zur näheren Beschreibung des Modells Kinzigtal und seiner ökonomischen Ausgestaltung: Hermann et al, 2006

25 Gesetz über den Verkehr mit Arzneimitteln (letzte Änderung 01.08.2007)

26 Vgl. Krämer (2005)

27 Vgl. AOK Bundesverband (2007)

28 ebd.

29 Ärzte Zeitung vom 17.03.08; Seite 14; „Klinikbetreiber berichten über Fehler"

30 Vgl. Lauterbach/Lüngen/Gerber (2004); Lauterbach/Gerber/Lüngen (2004); Lauterbach/Gerber/Lüngen (2006); Lauterbach/Gerber/Stollenwerk/Lüngen (2006); Lauterbach/Lüngen/Gerber et al. (2007)

31 Vgl. Wille/Wolff (2006)

32 siehe 27

33 Vgl. Hildebrandt (2007)

34 Vgl. Düsing (2006)

35 Vgl. AOK Bundesverband (2007) 